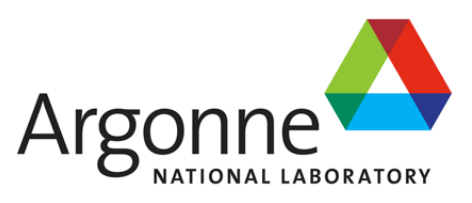

ANL-ART-238

\title{
Software Quality Assurance for EBR-II Fuels Irradiation and Physics Database (FIPD)
}

Nuclear Science and Engineering Division 


\begin{abstract}
About Argonne National Laboratory
Argonne is a U.S. Department of Energy laboratory managed by UChicago Argonne, LLC under contract DE-AC02-06CH11357. The Laboratory's main facility is outside Chicago, at 9700 South Cass Avenue, Argonne, Illinois 60439. For information about Argonne and its pioneering science and technology programs, see www.anl.gov.
\end{abstract}

\title{
DOCUMENT AVAILABILITY
}

Online Access: U.S. Department of Energy (DOE) reports produced after 1991 and a growing number of pre-1991 documents are available free at OSTI.GOV (http://www.osti.gov/), a service of the US Dept. of Energy's Office of Scientific and Technical Information.

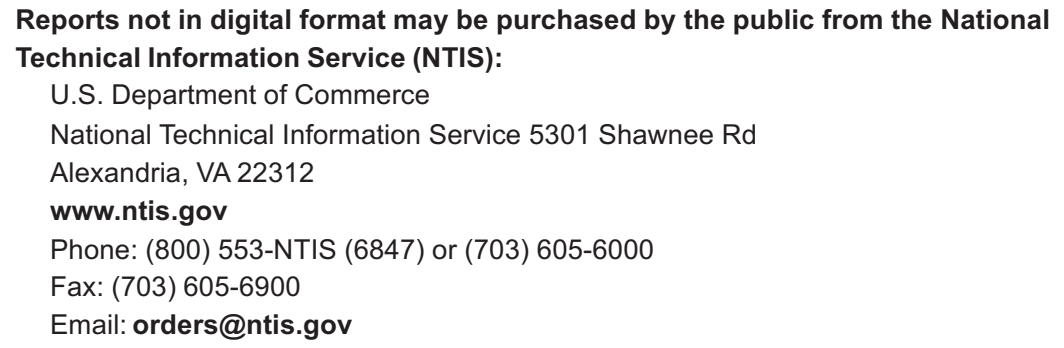

Reports not in digital format are available to DOE and DOE contractors from the Office of Scientific and Technical Information (OSTI):

U.S. Department of Energy

Office of Scientific and Technical Information

P.O. Box 62

Oak Ridge, TN 37831-0062

www.osti.gov

Phone: (865) 576-8401

Fax: (865) 576-5728

Email: reports@osti.gov

\section{Disclaimer}

This report was prepared as an account of work sponsored by an agency of the United States Government. Neither the United States Government nor any agency thereof, nor UChicago Argonne, LLC, nor any of their employees or officers, makes any warranty, express or implied, or assumes any legal liability or responsibility for the accuracy, completeness, or usefulness of any information, apparatus, product, or process disclosed, or represents that its use would not infringe privately owned rights. Reference herein to any specific commercial product, process, or service by trade name, trademark, manufacturer, or otherwise, does not necessarily constitute or imply its endorsement, recommendation, or favoring by the United States Government or any agency thereof. The views and opinions of document authors expressed herein do not necessarily state or reflect those of the United States Government or any agency thereof, Argonne National Laboratory, or UChicago Argonne, LLC. 


\section{Software Quality Assurance for EBR-II Fuels Irradiation and Physics Database (FIPD)}

prepared by

Micheal A. Smith, Lander Ibarra and Aaron Oaks

Nuclear Science and Engineering Division, Argonne National Laboratory

September 30, 2021 


\begin{abstract}
The Fuels Irradiation and Physics Database (FIPD) is an ongoing DOE project on archival of the EBR-II metal-alloy fuel irradiation experiments. As part of its use in support of license applications, the Quality Assurance Program Plan (QAPP) was drafted and endorsed by NRC in an effort to demonstrate its compliance with regulatory expectations. Software Quality Assurance (SQA) for the physics portion of FIPD is intended to qualify the calculated quantities such as fuel and cladding temperatures, neutron fluence and axially varying burnup estimates for irradiated fuel elements. This report covers the initial evaluation of SQA status of three neutron physics and thermo-fluid codes (REBUS, RCT and SE2RCT) that form the basis of calculated quantities for as-irradiated characteristics of the tested metallic fuel elements. The report also introduces and SQA plan to address the identified deficiencies.
\end{abstract}

The REBUS, RCT, and SE2RCT codes are all part of the Argonne Reactor Code (ARC) code system. There is considerable knowledge and experience on REBUS and RCT but relatively less on SE2RCT. During FY2021, efforts focused on an assessment of how the data in the EBR-II Physics and Analysis DataBase (PADB) is generated with SE2RCT and used in FIPD. Additional tasks included considerations of uncertainties for power estimates in REBUS and RCT calculations and their impact on the combined RCT methodology. The RCT software usage in FIPD was assessed this year and the input/output details studied. A "requirements" document was created that identifies the key features of the RCT software being used in FIPD that need to have SQA documentation. A brief discussion on the history of RCT and its input is included in this report along with the basic SQA roadmap laid out in the requirements document.

The SE2RCT software usage in FIPD is still being studied noting that there is no current manual. As part of the work done this year, two bugs were identified in the SE2RCT software which have a minor impact on the accuracy of the results it produces. No requirements document has been created, but one identified feature of SE2RCT being used that needs verification was its fuel pin temperature calculation. The work completed this year confirms that the approximations which will be included in the software verification report for SE2RCT are accurate.

In addition to software quality assurance work for RCT and SE2RCT, an automated verification framework is proposed to simplify the software quality assurance process. The purpose of this framework is to streamline code verification and documentation while minimizing repetitive tasks for code developers and reviewers. The reduction of repeated input (between reference solution, software, and documentation input) throughout the SQA process reduces potential for human errors during the preparation of the supporting software quality records. The automation of the verification and documentation process proposed for this project leverages the existing verification structure already in place for the SAS4A/SASSYS-1 code. 


\section{TABLE OF CONTENTS}

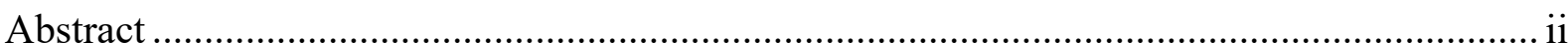

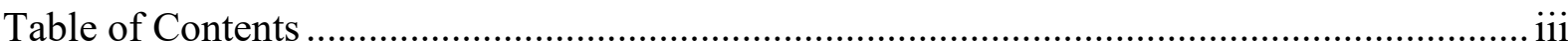

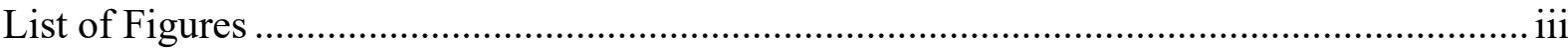

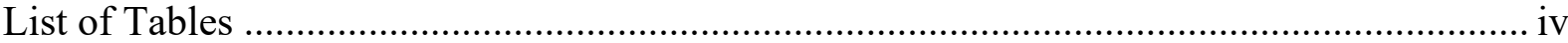

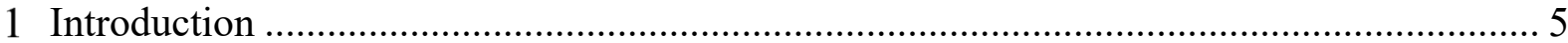

2 Overview of the Quality Assurance Program of the SFR Metallic Fuel Database (FIPD) ... 6

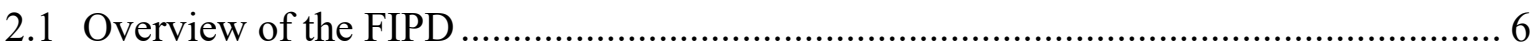

2.2 Role of FIPD in the Argonne Reactor Code (ARC) code system................................. 7

2.3 Argonne QAPP for SFR Metallic Fuel Data Qualification........................................... 9

2.4 FIPD Quality Assurance Program Overview ………………………………………. 10

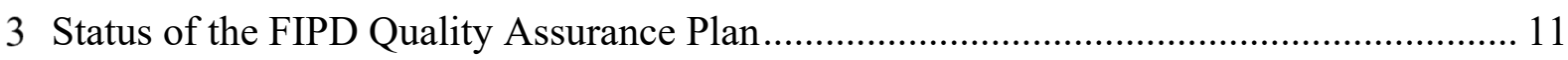

3.1 Data Qualification - Assessing Uncertainty in the PADB ………………………......... 11

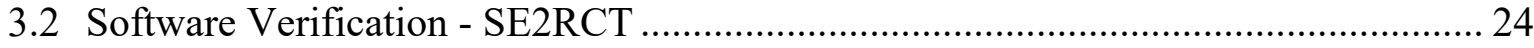

3.2.1 Identification and Correction of Coding Errors in SUPERENERGY-2 _........... 25

3.2.2 Verification Work - Pin calculation Routine ..................................................... 28

3.3 Software Verification - Automated Code Verification and Documentation................. 32

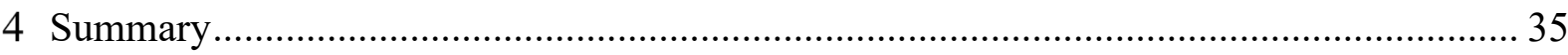

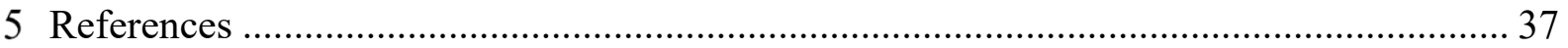

\section{LIST OF FIGURES}

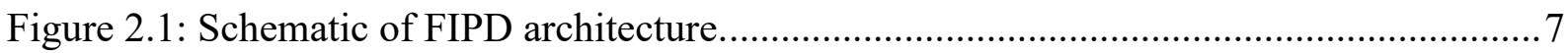

Figure 2.2 ARC Connected Code Suite for Fast Spectrum Reactor Analysis ............................ 9

Figure 2.3: SFR Data qualification planning and implementation structure. ............................ 10

Figure 3.1 EBR-II Criticality Prediction Calculated with REBUS ..........................................12

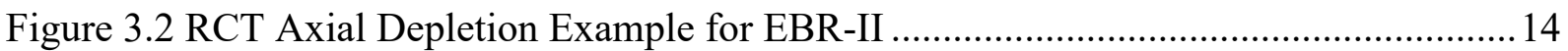

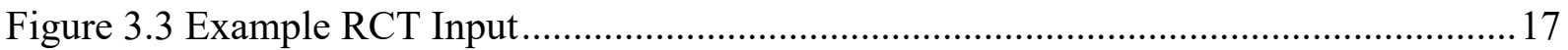

Figure 3.7. An Example of the RCT Flux and Current Output Table....................................2

Figure 3.8. An Example of the Average Nuclide Densities, Burnup, and Fluence Table ........22

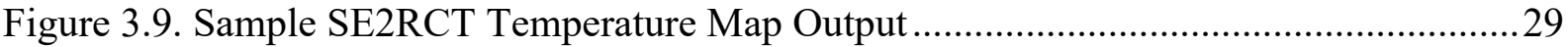

Figure 3.10. Results for the Fuel Pin Temperature Calculation.............................................. 31

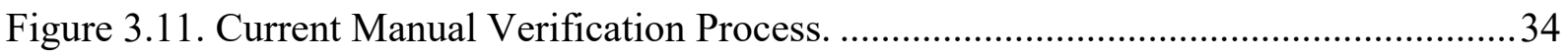

Figure 3.12. Proposed Verification Process ................................................................................ 35 


\section{LIST OF TABLES}

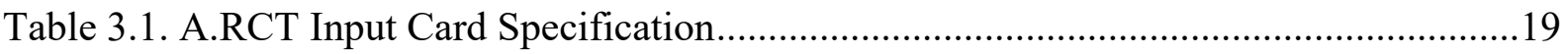

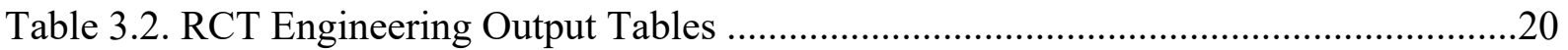

Table 3.3. RCT Identified Verification Tasks......................................................................23

Table 3.4. Fuel Centerline Results for the Fuel Pin Temperature Calculation ..........................32

Table 3.5: V\&V Test Suite Development Methodology .........................................................33 


\section{Introduction}

Qualification efforts of the legacy metallic fuel data generated during operation of US sodium cooled fast reactors (SFR) is supported by the DOE office of Nuclear Energy (DOE$\mathrm{NE}$ ). The effort aims to evaluate the pedigree of the data generated during the program and to support the use of the qualified data for licensing of advanced reactor designs which utilize metallic fuel. Other applications of the qualified data include its use in supporting modeling and simulation activities and fuel performance code development, as well as other end users including other DOE programs (e.g., NEAMS) and educational institutions. The legacy metallic fuel data were collected at the EBR-II and FFTF reactors during their operations starting in the early 1960's for EBR-II and 1984 for FFTF until their shutdown. This experience generated data for both metallic and oxide fuels. In particular, U-Zr based metallic alloy fuel data were generated during the Integral Fast Reactor (IFR) program (1984-1994), and are currently maintained in the DOE-NE supported Fuels and Irradiation Database (FIPD) [1].

FIPD is an online archive of irradiation experiments conducted at the Experimental Breeder Reactor II (EBR-II) [2-7]. The database, available at https://fipd2.ne.anl.gov/, provides means to search for details of specific irradiation experiments conducted in EBR-II. The FIPD program started to formulate its QA procedures in 2016-2017 and it led to development of the Quality Assurance Program Plan QAPP [8] by Argonne National Laboratory (herein referred to as Argonne). The purpose of the QAPP is to provide procedural guidance for qualifying these legacy metallic fuel data. The QAPP uses the standards of ASME NQA-1-2008/2009. In some cases, specific provisions and measures have been prepared to meet some aspects of 10CFR50 Appendix B, "Quality Assurance Criteria for Nuclear Power Plants and Fuel Reprocessing Plants." The NRC has recently reviewed the QAPP and determined it satisfies the quality assurance requirements of Appendix B to Title 10 of the Code of Federal Regulations (10 CFR) Part 50 and has endorsed the plan to support future license applications.

The plan introduces the following personnel requirements:

1) Senior management: Defines the expectations and goals for the project and ensures that groups A-C are identified with proper funding to maintain the activity.

2) Group A: Formed by the staff assigned with specific tasks for the project. For FIPD QA, this includes tasks can include data recovery from past reports, performing calculations, conducting software verification, and documenting the results of these tasks.

3) Group B: Formed by the staff assigned to review the work being done by Group A is correct and properly documented. For each task, the group B person should be knowledgeable, but independent of the actual task being done.

4) Group C: Formed by the staff responsible for creating the NQA-1 procedures and for verifying that groups A and B are carrying out the work consistent with the procedures. They coordinate with senior management and can be considered the management layer of the QA program.

Up to this point, all the work done in FIPD can be considered work done by Group A. As such, it must be processed by Group B. As part of this year's work, FIPD has identified members of all four groups cited above and effort is being put into reviewing and updating the past work with necessary procedures in place for conformance with QAPP. All remaining details on the 
setup activities QAPP implementation will be reported in the compendium FIPD deliverable report.

A key element of software quality assurance includes determination of software acceptance with respect to key critical characteristics. These critical characteristics define the features, capabilities, and attributes a software must be able to demonstrate with some level of confidence over a prescribed range or operating envelope. For the purposes of a licensing, critical characteristics by which a software will be measured will primarily relate to the software's ability to model specific phenomena. To demonstrate software adequacy verification and validation is performed. Qualification of data in the EBR-II Physics and Analysis DataBase (PADB) and verification of software relying on this database is provided in Section 3.

The remainder of this document is structured as follows. Section 2 provides background on the FIPD structure, an overview of the importance of the fuel database for the Argonne Reactor Code (ARC) code system, and the corresponding quality assurance requirements relevant to software dedication and qualification requirements. An overview of an automated verification framework is proposed in Section 3 to simplify the software quality assurance process. Lastly, the conclusions of this effort and a path forward are identified in Section 4.

\section{Overview of the Quality Assurance Program of the SFR Metallic Fuel Database (FIPD)}

\subsection{Overview of the FIPD}

The FIPD contains a broad range of content relating to the Integral Fast Reactor (IFR) program metallic fuel experiments (Figure 2.1), covering pin-level operating conditions calculated using a variety of physics codes, reactor-level operating data, digitized versions of post-irradiation examination (PIE) data, and an extensive document library. 


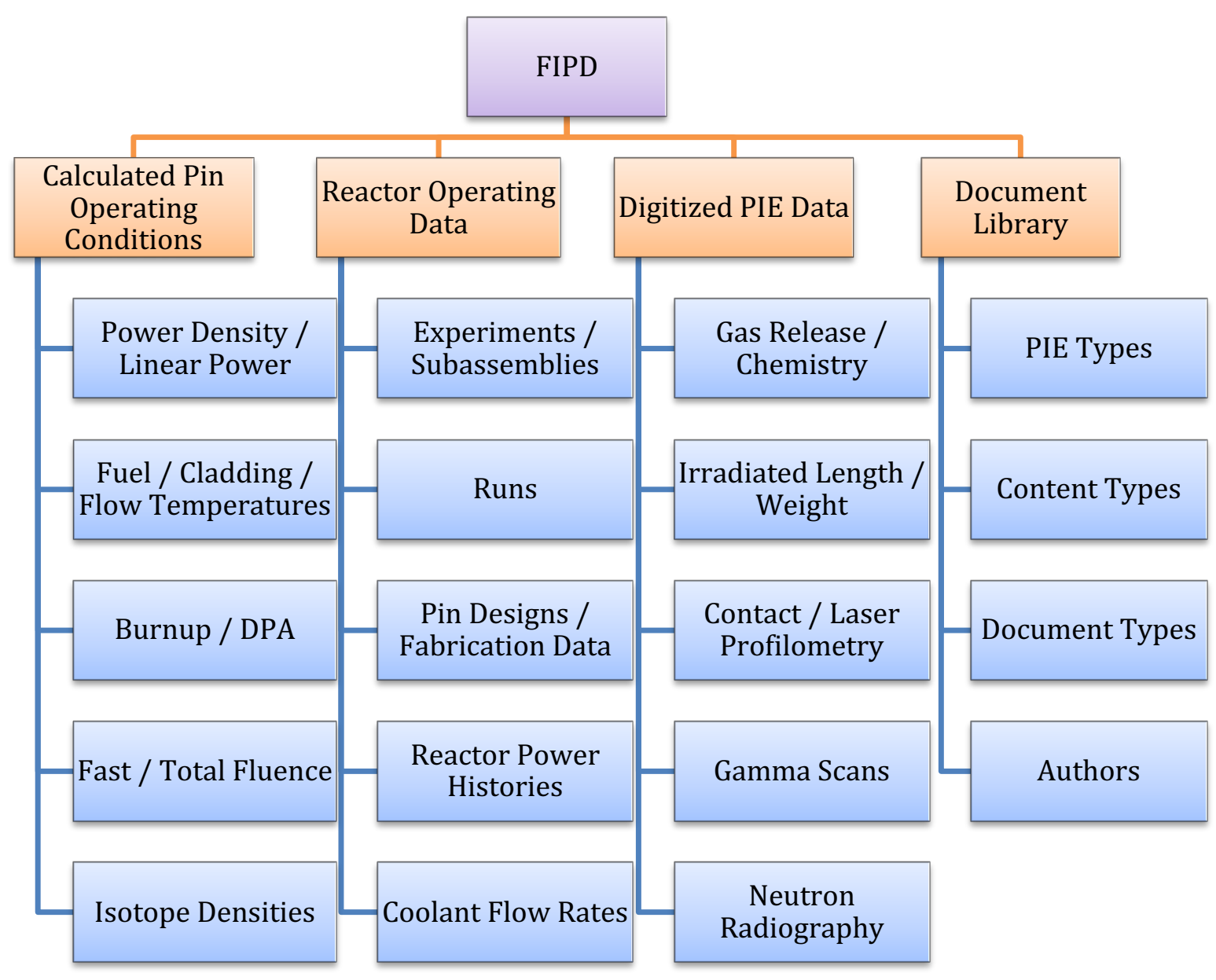

Figure 2.1: Schematic of FIPD architecture.

FIPD currently stores the data from 32 experiments. Multiple types of records pertaining to the different steps of conducting an experiment at EBR-II are accessible in the FIPD including: test specifications, data package, fabrication records, QA records, post irradiation examination (PIE) data generated at AGHCF (Alpha Gamma Hot Cell Facility) or HFEF (Hot Fuel Examination Facility), and operating conditions records.

\subsection{Role of FIPD in the Argonne Reactor Code (ARC) code system}

As part of the FIPD database construction, both measured and calculated data are used. Direct instrumentation of individual assemblies to assess the power distribution or flow rate was not practical in EBR-II although indirect means were available to infer what these were to some degree. To provide this information, various parts of the Argonne Reactor Code system (ARC) were used [10-23] to produce a calculated value of the missing information. The data source for the ARC software is the Physics Analysis DataBase (PADB) which was organized in 1994 to support the IFR fuel cycle demonstration [4].

The PADB contains the operational loading details of the EBR-II core and its purpose in the IFR program was to provide fuel cycle analysis support for the $\mathrm{Zr}$ alloyed uranium metallic fuel form being considered for IFR. The PADB includes a history of the core loading for many 
operational cycles of EBR-II giving the assembly geometry and fuel composition, control rod positions, power level for each cycle such that contemporary software could be used to carry out fuel depletion calculations. There is a considerable amount of measured data and calculated data in PADB. As such, data validation is challenging due to the complexity to characterize uncertainty propagation.

The PADB data was organized with respect to different EBR-II runs (operational periods in which no fuel was loaded into or out of the core), starting from Run 130A to Run 170B where EBR-II was shut down at the end of Run 170B. Run 130A to 170B accounts for the ten-year time period of EBR-II operations from 1984 to 1994 . The targeted fuel form was first loaded at the beginning of Run 133A, and before this run, uranium-fissium fuel was used. The detailed fuel and operational data were available in the PADB only after Run 130A, which was a few runs before Run 133A, to introduce some margin before the first metallic fuel subassembly was loaded into the EBR-II core. Excluding fresh fuel, all compositions for all fuel assemblies are based upon the fuel cycle analysis capabilities at the time.

The intention of the FIPD program was to treat the PADB as fixed data such that it did not have to be reviewed as part of the NQA-1 procedure. This proved problematic as numerous problems were identified in PADB and the various ARC codes used to generate the calculated results were identified to have bugs in them which altered the calculated results. In several cases, the updated calculation capabilities of ARC were used to provide data that has been directly loaded into FIPD to replace erroneous earlier data. While the ideal goal of the NQA-1 aspect of FIPD is to focus only on the recovery of the measured data, the inclusion of the calculated data poses a problem for QA and thus determining the necessary software QA envelope is an active part of work in the NQA-1 setup for FIPD. At present, the RCT [21] and SE2RCT [24] parts of ARC have been identified as the minimum software components in the QA envelope and thus part of this year's focus was on assessing that software. Figure 2.2 shows the connected set of codes in ARC for typical fast spectrum reactor analysis. For FIPD, SE2RCT is similar to SUPERENERGY [19] but it is directly connected to RCT. In that regard, there is no connection with SAS4A/SASSYS-1 [29], NUBOW [23], PERSENT [20], or GAMSOR [18] as those components are not needed in FIPD. 


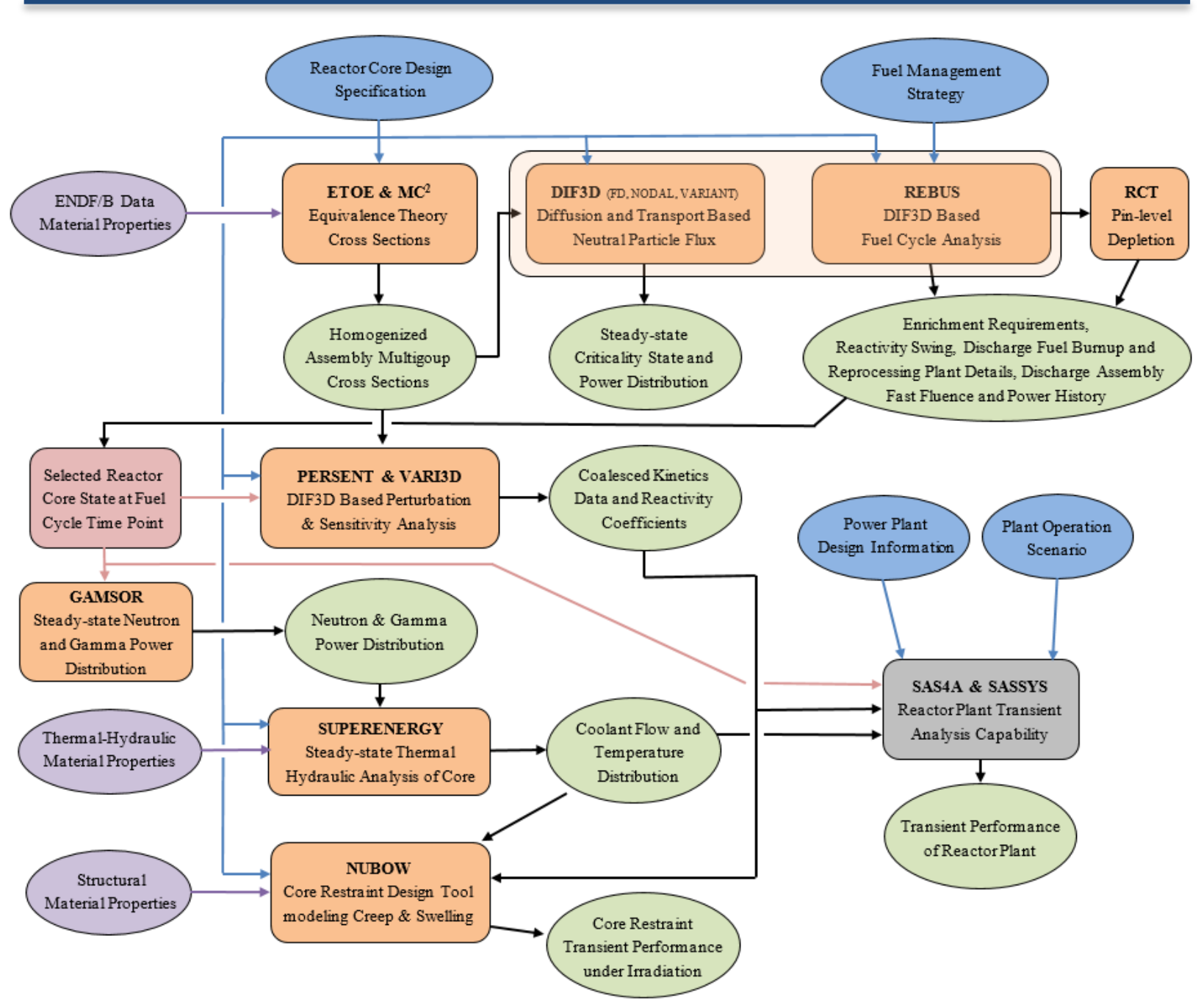

Figure 2.2 ARC Connected Code Suite for Fast Spectrum Reactor Analysis

\subsection{Argonne QAPP for SFR Metallic Fuel Data Qualification}

Managing the amount of data in the FIPD database generated during the US IFR program presents a significant challenge for retaining data integrity and availability. The American Society of Mechanical Engineers (ASME) Standard NQA-1 (NQA-1) 2008/2009 Addenda provides appropriate requirements to assess data integrity, completeness, and availability. Accordingly, Argonne established a Quality Assurance Program Plan (QAPP) for the SFR Metallic Fuel Data in FIPD using the ASME NQA-1 2008/2009 Addenda standard [8]. This QAPP is the top-level policy document that establishes the way in which quality is achieved in the SFR legacy fuel data. Implementing documents prescribe more detailed responsibilities and requirements and define the organizational interfaces involved within the scope of the QAPP. The Quality Assurance and Vendor Inspection Branch (IQVB) in the Nuclear Regulatory Commission (NRC) has completed its review and determined that the Argonne QAPP for SFR Metallic Fuel Data Qualification satisfies the quality assurance requirements of Appendix B to Title 10 of the Code of Federal Regulations (10 CFR) Part 50. The implementation of this QAPP is essential to ensure quality of data in FIPD, and it will be one of the key efforts in the ongoing FIPD development. In addition to the plan to qualify the data, another plan has been 
developed to qualify the software associated with the database. This plan is designated the Nuclear Fuel Codes and Data (NFD) Software Quality Assurance Program Plan (SQAPP) as presented in [9]. The relation for QAPP and SQAPP for metallic fuel data qualification can be seen in Figure 2.3. More details about SQAPP can be found in references [8] [9].

\subsection{FIPD Quality Assurance Program Overview}

The current QA program aims mainly at supporting the industry efforts for licensing advanced SFR designs that utilize metallic fuels. In addition, other DOE programs or university users for R\&D purposes may have different qualification requirements for the metallic fuel data. The differences between types of data needed for each end user is shown in Figure 2.3: the industry users will require more detailed documentations, and all available records of the data, even if the qualification was performed using the peer review method, which is one of the qualification methods identified in reference [8]. This is compared to the needs by other end users such as the Nuclear Energy Advanced Modeling and Simulation (NEAMS) program for modeling and simulation or non-NRC based qualifications, as well as universities R\&D activities. For those end users, the data needs are focused on the PIE data, operating parameters, and fabrication data for the experiments.

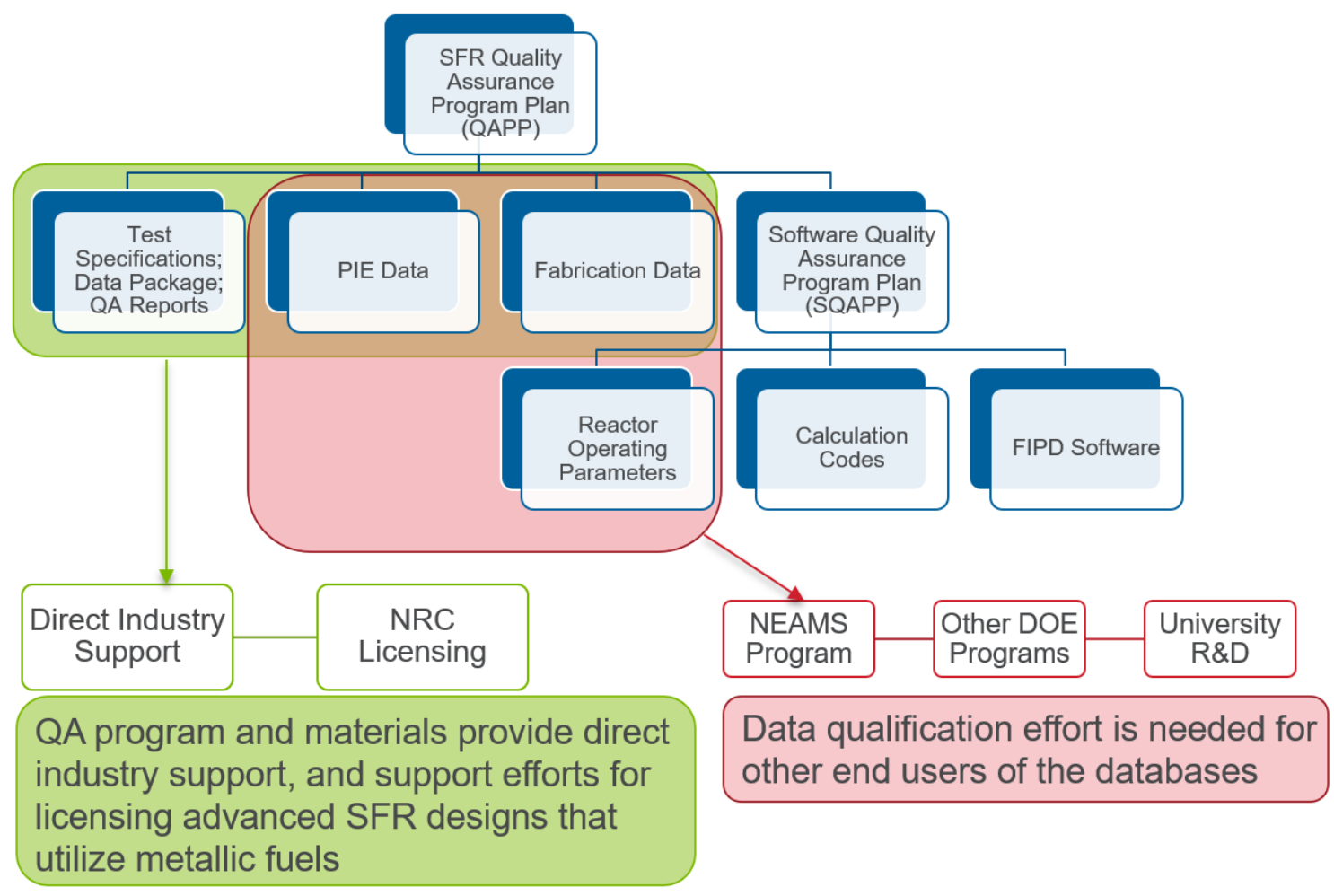

Figure 2.3: SFR Data qualification planning and implementation structure. 


\section{Status of the FIPD Quality Assurance Plan}

\subsection{Data Qualification - Assessing Uncertainty in the PADB}

Much of PADB provides the necessary information to run the 10-year depletion period, but no details on the uncertainty of the provided data was given. This is partly because the operational history of a real reactor is complicated and the modeling purpose of PADB was intended to be simple. As an example of missing uncertainty, we consider the power level here.

In PADB, the power for each depletion step is given as a single value for the entire step inferring that this was the average power. Power in a fast reactor is not easy to directly measure and from the literature, EBR-II had several ways of estimating the operating power. There were at least three separate approaches to infer the power level of EBR-II and the actual source(s) of the reported power level in PADB is not given. The most accurate power measurement technique used bulk inlet and outlet coolant temperature measurements and the inlet mass flow rate to compute the total energy being produced by the reactor. Periodically, this power measurement had to be recalibrated as there was some drift in the measurement equipment (thermal couples).

Over time, some of the measurement equipment failed and thus the power estimation would then be primarily based upon one of the other techniques. Because each operational period in EBR-II was documented in a run report, this is mostly likely the source of the average power that appears in PADB which was processed in some manner to construct an average over the time period. Most likely the power reported in the run report was the average of the available measured approaches. As stated, there is no uncertainty provided on the power level in the PADB database but it should be clear that it was not more than $99 \%$ accurate for any given depletion step.

Further complicating the power aspect is that the ARC software calculations are not exact. For depletion in particular, the power level and its distribution are two problematic issues identified for the FIPD usage of the ARC modeling software. The power normalization in REBUS [12-13] and RCT [21] is based upon a single level KERMA factor that is independent of the spectrum and all energy from a given reaction is assumed to be absorbed at the point of the reaction. For fissioning actinides such as U-235, this approach is very accurate for estimating the total power delivered due to fission. For other neutron capture reactions it can be quite poor as the KERMA factor used is taken from a thermal spectrum assumption rather than the local fast spectrum in EBR-II.

Other accuracy concerns exist with the REBUS approach such as the physical location of where the energy is deposited as gamma emission with the single level factor assumes the energy is deposited in the assembly generating the gammas where the gamma rays move throughout the domain. The GAMSOR part of ARC was constructed correct the gamma transport and improve the single level KERMA factor approach for the power distribution in ARC. In GAMSOR, MC2 [11] is used to produce energy dependent KERMA factors for neutrons and gammas, and thus spectrum specific, and the DIF3D code is used on a given time point from REBUS to carry out the neutron transport and gamma transport throughout the domain. From experience, the total energy production for a reactor from GAMSOR has been observed to be as much as 5\% in error from the REBUS prediction and for EBR-II it was found to be $5 \%$. Given GAMSOR should be more accurate, this indicates that the REBUS/RCT power 
level being used can easily lead to a miss-prediction of the true burnup, especially over a ten year period. Further, the lack of considering gamma redistribution in REBUS can lead to a missprediction in the power distribution by assembly and thus the temperature distribution in the follow-on SE2RCT calculations although this is likely of less concern for FIPD.

To understand the impact of a mistake in power, Figure 3.1 is provided as an example of the criticality prediction (keff) over the 10 -year operational period. To begin, at each time point, EBR-II was critical $(\mathrm{keff}=1.0)$ and thus the figure should provide solutions that are reasonably close to critical at all-time points. As can be seen, in the early part of the 10-year period, keff is nearly $1.5 \%$ low and there is a positive drift such that by the end of the operational period the calculational results are close to critical. REBUS is a deterministic code and when combined with the cross-section data errors, it is common for a model of a critical reactor to be up to 100$300 \mathrm{pcm}$ off from the measurement. For clean critical reactor problems such as ZPPR [25-26], DIF3D was observed to be within $200-300 \mathrm{pcm}$ on a wide range of reactor concepts and loadings. Thus, to be $1.5 \%$ off would indicate a serious error in the modeling methodology for EBR-II or a problem with the reactor model itself.

As stated earlier, the initial loadings are based upon calculated compositions of irradiated uranium-fissium drivers using the best available methodology at the time (e.g. 1994 capabilities). From the graph shown, it is very reasonable to assume that the burnup of those assemblies was overestimated in some manner to produce errors of this magnitude. Thus while the improved ARC capabilities today for depletion might improve those approximate calculations, using the erroneous starting data causes a propagating power distribution error as the power can be artificially higher in the higher worth fresh fuel being inserted due to a depressed worth of the preceding irradiated fuel that has more burnup than reality.

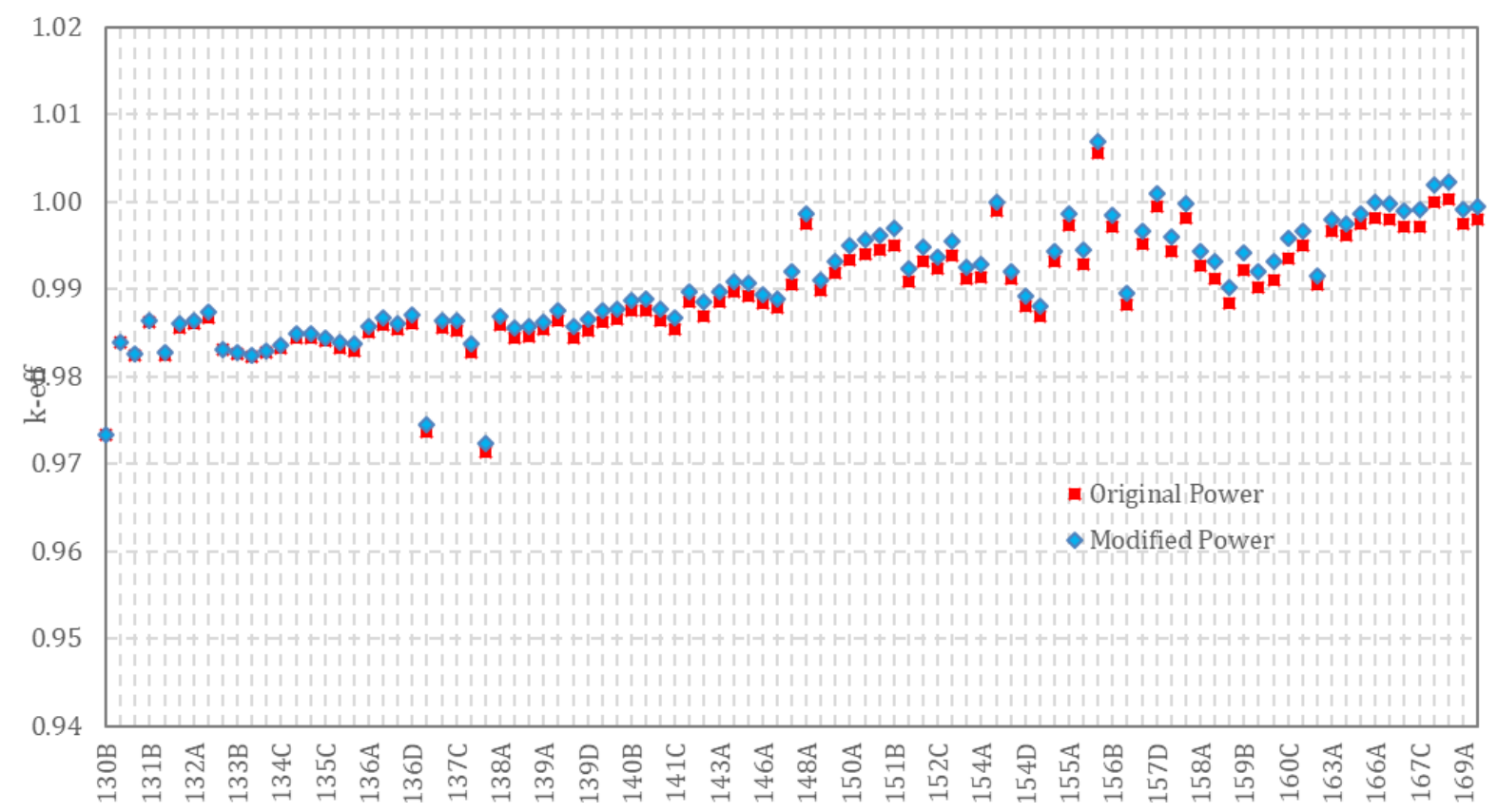

Figure 3.1 EBR-II Criticality Prediction Calculated with REBUS

In addition to the general drift in the criticality estimate, one can also see several time points where the data is not smooth such as between loading 136D and 137C. In these cases, the control 
rod positions that are included in PADB are believed to simply be inaccurate. EBR-II was rather unique in that it had regular control rods and high worth control rods. The high worth control rods were effectively movable driver assemblies and it was not uncommon to have the high worth control rods offset from the rest of the core to control the beginning of cycle (BOC) excess reactivity. This is a particularly difficult aspect of EBR-II for ARC to calculate well and it can alter the bias in keff from a conventional loading where the active core is all axially aligned. While this could be the source of error on these points (a $\sim 1 \%$ additional error in keff), the regular control rod positions contained in PADB could also simply be erroneous.

To better assess the impact of the KERMA related power normalization in ARC, the entire 10 -year period was completed with two power levels, one at the PADB specified level, and one with 5\% less power (modified power). As can be seen in Figure 3.1, the importance of the power level normalization is non-trivial at the end of the 10 -year period, but it is relatively small $(0.02 \%)$ compared with the overall positive drift and control rod position issues.

To continue the discussion on uncertainty, we can turn the focus on the fuel cycle methodology itself. The RCT code [21] was specifically built for the IFR program to better model and analyze the pin level depletion details of EBR-II. In several of the EBR-II operational periods, fuel pins were removed from previously irradiated driver subassemblies and placed into other subassemblies for further irradiation (higher burnup). In addition, EBR-II was a very leaky core configuration and subassembly rotation was another key feature of RCT that was required beyond REBUS to produce accurate fuel pin depletion results. RCT is built upon the DIF3D-Nodal [15] diffusion theory capability which allowed the users to model many fuel cycle analysis stages without concerns for spatial mesh refinement requirements as was typical of DIF3D-FD applications. Recent modifications to RCT [27] have allowed the DIF3DVARIANT solution to be incorporated and used by RCT in the VTR analysis effort which has been used by FIPD in some of their more recent contributions to the database.

To avoid an excessively large PADB and to ensure a reasonable computational time, the REBUS models of EBR-II in PADB assume 3 axial fuel depletion regions. For special pins in RCT, the spatial details of depletion are assumed to have an axially quadratic shape in each depletion mesh even though the actual DIF3D flux solution methodology might be using a 4th or 6th order polynomial representation. Figure 3.2 shows an example of one of the depletion predictions from RCT. In this example, the total Pu content is divided by the total Uranium to allow a relative error to be used rather than an absolute one between the measurement and the calculation. Also included is the normalized $\mathrm{Pu}$ and $\mathrm{U}$ results (with respect to the average content) for clarity on the distribution. 


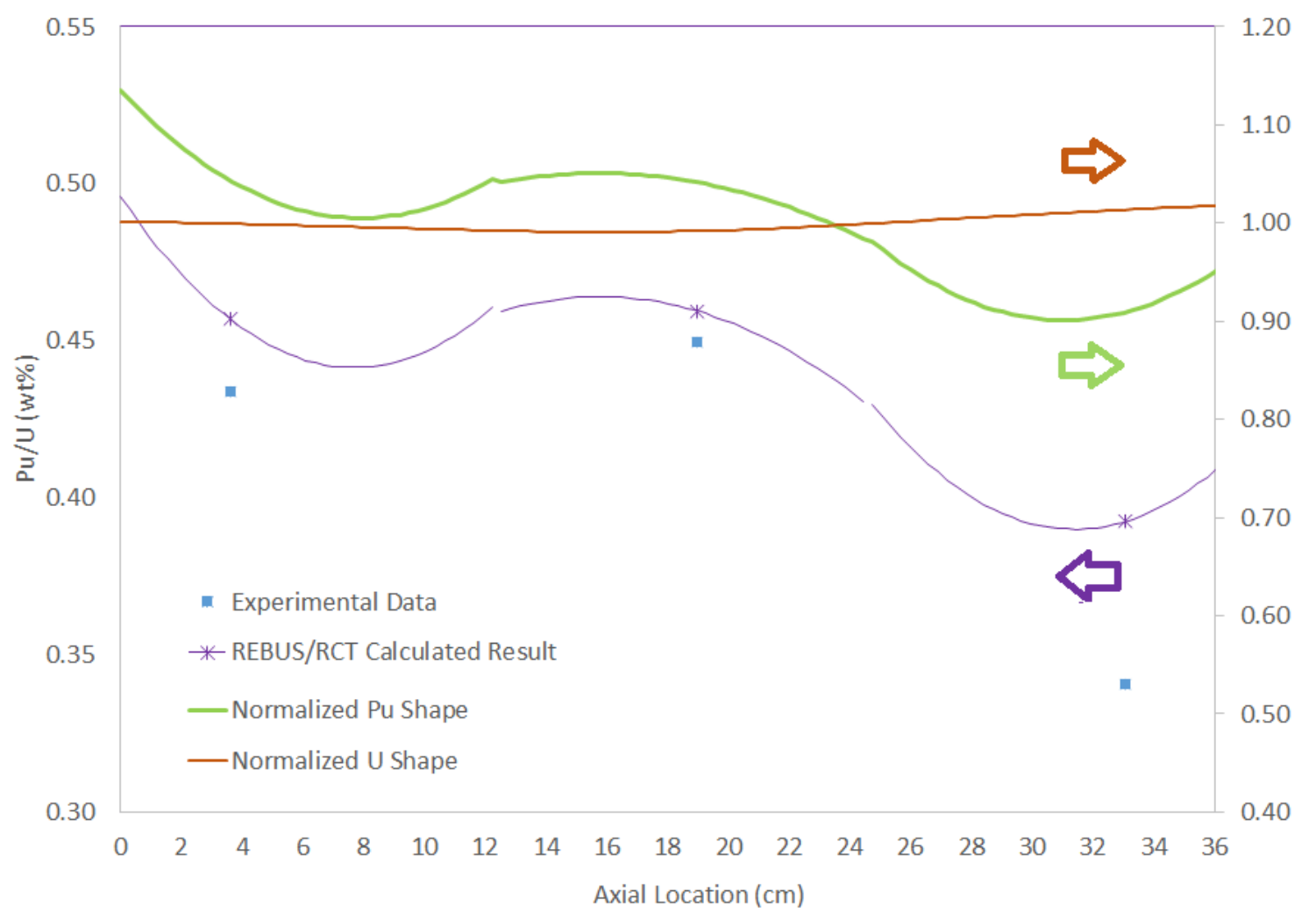

Figure 3.2 RCT Axial Depletion Example for EBR-II

In Figure 3.2, the measured data consists of samples from three chopped segments of a single pin in this assembly. From the measured data, one can infer a quadratic shape is present with a considerable tilt towards the bottom of the active core but a maximum still at the center of the domain. The tilt is expected due to the proximity of the lower reflector to the fuel which increases the power at the bottom of the fuel assembly considerably relative to the upper end of the active core. As a consequence, one expects an increase in the production of $\mathrm{Pu}$ and decrease in Uranium content relative to the upper part of the core as is seen. The three evaluation points with RCT also indicate a quadratic form, but the actual axial shape in the upper and lower axial meshes is rather non-physical. While the shape of the Uranium content is somewhat explainable, the $\mathrm{Pu}$ shape has strong discontinuities at the axial mesh points indicating a failure in the flux interpolation scheme along with the overall non-physical shape. Had the sample point been closer to the bottom of the fuel pin, it should be rather obvious that the RCT calculated results would have more error in it.

The source of this error is fundamental to the methodology of RCT which assumes the user would increase the number of axial depletion meshes to improve the calculation accuracy as is necessary. There are also concerns with the cross section generation methodology which might not be good enough to handle the strong interaction of the lower reflector with the adjacent fuel. Whether the above is a significant enough concern for FIPD to warrant a re-evaluation of PADB with more axial depletion meshes is not clear at this time. Based upon what data is being fed into FIPD, the above displayed RCT issues are likely not relevant as most of the FIPD measurements are focused on central samples from the pin. The power distribution issues 
discussed earlier that are fed into SE2RCT, are of concern and most likely the uncertainty in that power distribution would be rather useful.

The discussion above adequately summarizes the minor amount of work done this year to consider aspects of uncertainty in PADB and the impacts of how the ARC software is being used. This entire discussion is more appropriate for validation and was not a focus of the work in this project, but just a natural outcome of the involved staff working on two disconnected projects that dealt with validation of the ARC software on PADB. The reason it is relevant is that this work helped identify the way RCT and SE2RCT are being used for FIPD which is vital to determine the software verification needs of the software. Software Verification - RCT

RCT (ReConsTruction) is a follow-on utility program for the REactor BUrnup System (REBUS) fuel cycle analysis code and the built in DIF3D code. Both DIF3D and REBUS software verification must be completed before the present RCT work as those pieces of software underpin the actual accuracy of RCT. As part of the work done this year, the primary capabilities of RCT used by FIPD were identified and used to establish a set of RCT verification tasks. A 30+ page report was written detailing the RCT usage in FIPD and specific outputs from it were identified. That report is under review and when accepted, it will serve to define the set of verification work that needs to be done on RCT. A similar path was followed for the DIF3D software where its' requirements and verification tasks were established in [28]. In this section, only a summary of RCT and the verification work is provided.

REBUS has been maintained by Argonne since the early 1960s to support its reactor design mission. That software transitioned from the original REBUS to REBUS-2 in the mid-1970s and to REBUS-3 in the mid-1980s. Since then, REBUS has gone through many revisions to the current REBUS-11. Note that this version numbering is consistent with the progression of DIF3D, the base flux solver that REBUS is built upon. REBUS was designed around the DIF3D code and thus is primarily based upon a semi-structured grid geometry using the exact same binary interface files and variable naming scheme as in DIF3D. Of particular interest for FIPD is the ability of REBUS and RCT to perform depletion and fuel cycle operations on hexagonal 3D geometries consistent with the EBR-II experimental reactor operations data.

RCT was added to the ARC software system in 1993 and was specifically built to carry out fuel pin analysis for ongoing EBR-II measurements. In the EBR-II calculation process, each cycle (operational period within which no fuel shuffling was performed) is modeled as a single step REBUS fuel cycle calculation. REBUS obtains the average flux in each region and thus obtains the average transmutation of a given region (or mesh) over the cycle. RCT takes the assembly and pin design information as its input. The DIF3D-Nodal or DIF3D-VARIANT flux solutions are used to construct the flux distributions within in each DIF3D region (or mesh).

RCT manages the pin depletion in two approaches. In the first, a smooth interpolation basis is used in the assembly and the atom densities are cast into this basis and depleted. This approach will exactly reproduce the REBUS result and assumes a smooth distribution of starting pin isotopic atom densities. For pin level quantities such as atom density or flux, the basis is simply interpolated at each desired pin location. A normalization should be applied to guarantee that the average quantities are preserved for a given pin array discretization.

In the second approach, RCT introduces special pins. In this case, the flux solution is interpolated at the pin center, top, and bottom in each DIF3D mesh yielding a quadratic shape over the mesh. Depletion is carried out at those three points assuming the isotopic atom density 
concentration can be similarly defined. There is no normalization scheme in place to ensure that RCT will reproduce the REBUS result when using special pins and thus some amount of drift can occur. If the entire assembly was treated using special pins, then one could in theory renormalize the flux evaluation at all of the pin locations ( 3 points per pin) to ensure the REBUS result is preserved, but this is not done in RCT. Given the special pin treatment forms a bulk of the FIPD usage, considerable verification work will need to be done to assess the level of inaccuracy that this brings to FIPD as it is heavily used.

$\mathrm{RCT}$ stores the isotopic atom densities for the two schemes along with the power history in binary interface files which are needed as the input (BOC) to RCT and are produced as the output (EOC). Since REBUS can model the explicit fuel cycle of a given reactor, one should understand that for any given cycle, all previous cycles must be solved in order to obtain the solution for the desired cycle. Thus for each cycle, REBUS is used to obtain the EOC state followed by RCT to obtain the EOC pin level state.

As shown in Figure 2.2, RCT is not connected to the downstream components in the regular fast reactor analysis work. Instead, RCT output from a given cycle in PADB is directly taken as the input to SE2RCT which is not shown in Figure 2.2. SE2RCT itself is a modified version of Superenergy-2 to incorporate the pin power distribution from RCT and is discussed later in this manuscript. RCT itself makes almost no use of the other ARC software components as it is effectively a post-processing utility code for REBUS. One unique aspect of RCT compared with the other software in Figure 2.2 is its reliance on the REBUS created binary file RFILES. This file is intended to be used for restarting REBUS calculations and RCT commandeers it to extract the depletion chain details for any given REBUS calculation to avoid requiring the user provide any depletion chain input to RCT. RCT obtains composition details from the binary files generated by REBUS and thus it should be apparent that PADB contains all of the information necessary to build the REBUS model of each EBR-II depletion step in the 10-year period.

Given these details of how input is obtained by RCT, the actual user input for RCT is clearer, an example of which is given in Figure 3.3. Table 3.1 lists the card type input specification for RCT. In Table 3.1, the Card Type indicator "2.3" indicates the third parameter of Card Type 2. Because all of these card types are used by PADB, all of them must be verified as part of the FIPD software QA work. 


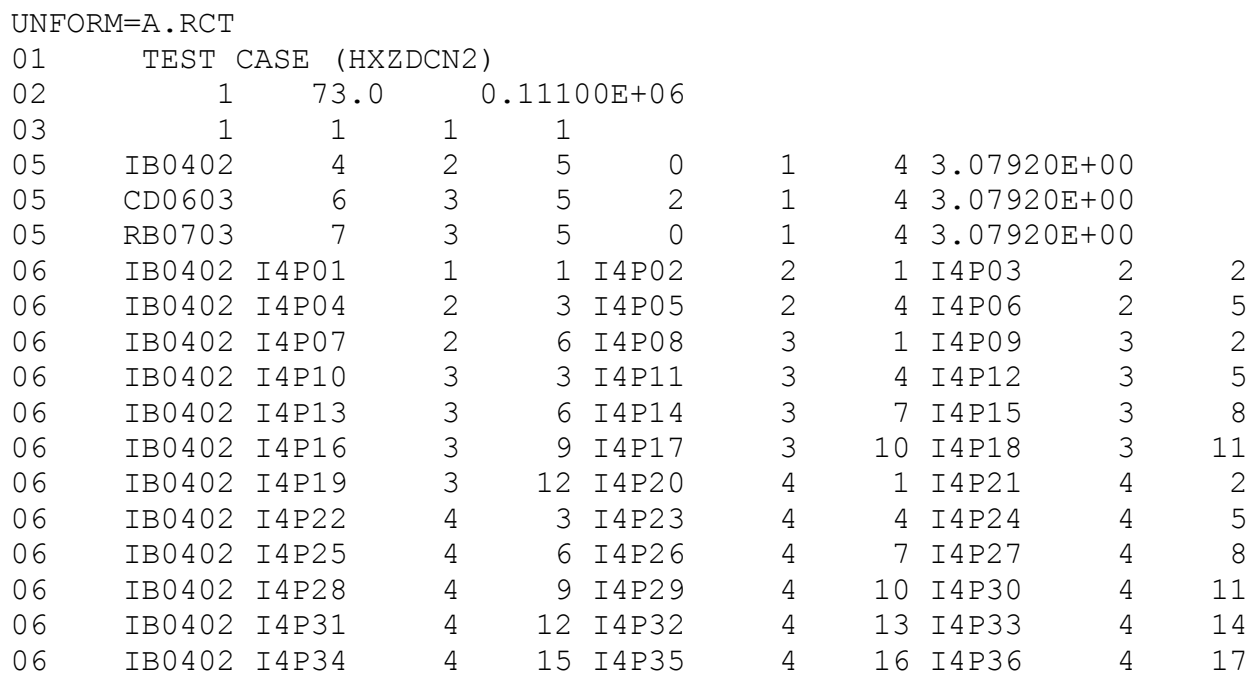

Figure 3.3 Example RCT Input

For brevity, the bulk of the RCT input cards are not covered here. Of particular note are Card Type 06, 07, and 08. In commercial reactors, it is not uncommon to have differences in the pins of an assembly (different enrichment, burnable poison, etc...). In EBR-II, several experimental assemblies were composed of pins with different fuel content and this input feature is used to separate the compositional impact in RCT as REBUS simply treats the entire assembly as a homogeneous region. Another operations aspect of EBR-II was the practice of taking irradiated fuel pins from one assembly and placing them into a different assembly to study the impact of higher burnup. In this manner, Card Type 06 allows the user to specify special pins in each assembly and their lattice position. Card Type 07 allows the user to specify the composition for each special pin. Finally Card Type 08 allows the user to link the special pin with the provided materials (e.g. use the same material for 20 different pins) and what subset of the axial range the special tracking is to be followed. The point of this discussion is to indicate that the complexity of a RCT input can become difficult to manage by hand and thus PADB was setup to control the entire process.

The RCT code can produce a considerable amount of output which can mostly be identified by the table headers in the RCT output. Table 3.2 summarizes the table headers and the creation of each table is controlled by the A.RCT input itself and thus some RCT calculations may not contain the stated tables in the output. Figure 3.4 provides an example of the seventh table type listed in Table 3.2. In this table, the flux solution at the corner points and surfaces are given along with the partial currents on each surface. As stated above, instead of treating the fuel pin depletion as a pin level aspect, RCT was designed to deplete assembly using a set of basis functions assuming a smooth interpretable function over the domain. Because of the use of DIF3D-Nodal and DIF3D-VARIANT, this approach makes sense as both have a fixed spatial polynomial approximation within each assembly. Taking this approach makes the use of DIF3D-FD next to impossible and with DIF3D-Nodal, a "tricky" interpolation scheme is introduced to obtain the corner point fluxes. While DIF3D-VARIANT does not need this as it constructs a full 3D basis, it also uses the interpolation scheme as it is embedded into RCT. Because the manual states that using this interpolation scheme near the outer domain boundary 
is problematic, this aspect will also require additional verification work as the magnitude of its impact is not stated.

The listed flux values in Figure 3.4 are used to define a interpolation rule over the entire hexagon. For depletion, the composition is factored into solutions at each flux point (six corners, eight surfaces, and one average value) as shown in Figure 3.5 which is an example of the ninth table type in Table 3.2. By taking this approach, the depletion system is solvable independently for each flux point rather than each fuel pin. Whether this is wise or not is not clear as there is no methodological literature review of the approach. By isolating and tracking the average value for the assembly, we know this approach should ensure that the interpolation preserves the REBUS result.

In contrast to this scheme is the introduction of special pins via the card type 06,07 , and 08 mentioned above. In these assemblies, the isotopic number densities are defined specific to each pin (top, bottom, and average). The flux values at each pin are taken from the interpolation rule based upon the average, corner, and surface flux values to obtain a top, bottom, and average flux value for each pin. The depletion calculation is then applied to each of the three positions to give atom densities at the top, bottom, and average of each fuel pin.

With the special pin approach, there is the potential for the methodology to not maintain the average flux and thus the depletion result from REBUS. This is primarily because of the decision to continue to use the interpolation rule-based depletion scheme for the remainder of the assembly which invariably is subjected to the average flux over the assembly and does not exclude the flux component applied to the individual pin(s). If one were to switch entirely to a pin level depletion, then one would expect that the average pin would interact with the average flux in the assembly and thus the average depletion result from REBUS would be maintained. However, this is also not the case and a normalization of the array of pin fluxes in each group is required to ensure that the pin fluxes produce the average flux result and thus average depletion result. Without this, there is significant potential for the special pin approach to drift away from the REBUS calculated approach upon which it is based.

From the above discussion, it should be clear that the verification work on RCT will be time consuming. The recent software verification work for REBUS took almost a year of effort and one can expect RCT to be longer due to the custom approach to define the interpolation rule and its associated depletion along with the special pin treatment. The identified work thus far can be broken into tasks as shown in Table 3.3. Categories are used to try and group the work into a common purpose. The focus of the first category is on the accuracy of the user input and will be collectively verified by all of the verification problems created. In the second category, the derived quantities used for the depletion interpolation are checked. Categories 3 and 4 are going to pose the bulk of the verification work as they require the depletion equations to be solved for a given data point. As stated, task c of category 4 is likely to fail as the special pin details are not imposed to reproduce the REBUS result. The last category was added to account for the PADB usage of RCT which is an aspect of FIPD and how RCT is used. 
Table 3.1. A.RCT Input Card Specification

\begin{tabular}{|c|c|c|c|}
\hline $\begin{array}{l}\text { Card } \\
\text { Type }\end{array}$ & Summary & $\begin{array}{l}\text { Card } \\
\text { Type }\end{array}$ & Summary \\
\hline 1 & Problem title to appear on page headers & 6 & Special Pin specification \\
\hline 2 & Problem Options and Parameters & 6.1 & Assembly name \\
\hline 2.2 & $\begin{array}{l}\text { Interface data set sentinel to take data from } \\
\text { STACK or individual files }(0 / 1)\end{array}$ & $6.2,6.5,6.8$ & Special pin name \\
\hline 2.3 & Refueling time (days) & $6.3,6.6,6.9$ & Pin lattice ring number for the unique pin \\
\hline 2.4 & Fast flux energy threshold $(\mathrm{eV})$ & $6.4,6.7,6.10$ & Pin lattice position along ring for the unique pin \\
\hline 3 & Edit Options & 7 & Special Pin Material Specification \\
\hline 3.1 & Flag to output nuclide densities $(0 / 1)$ & 7.1 & Material name \\
\hline 3.2 & Flag to output flux details $(0 / 1)$ & $7.2,7.4,7.6$ & Isotope name \\
\hline 3.3 & Flag to output power details $(0 / 1)$ & $7.3,7.5,7.7$ & Isotope atom density (atoms $/ \mathrm{cc} \cdot 10^{-24}$ ) \\
\hline 3.4 & Flag to output burnup details $(0 / 1)$ & 8 & Assignment of special pin to material \\
\hline 4 & Files to be read from the stack file & 8.1 & Material name \\
\hline 4.1 & Binary file name (ZNATDN, LABELS, etc...) & $8.2,8.5,8.8$ & Special pin name \\
\hline 4.2 & Version of the binary file & $8.3,8.6,8.9$ & Starting axial burn region number \\
\hline 4.3 & Starting record position in the STACK file & $8.4,8.7,8.10$ & Ending axial burn region number \\
\hline 5 & Specification of Assemblies for Reconstruction & 9 & Initial heavy metal densities of assemblies \\
\hline 5.1 & Name to assign the assembly & $9.1,9.5$ & Assembly name \\
\hline 5.2 & Hex ring position of the assembly & $9.2,9.6$ & Starting axial burn region number \\
\hline 5.3 & Ring position for the assembly & $9.3,9.7$ & Ending axial burn region number \\
\hline 5.4 & Number of axial burn regions in REBUS & $9.4,9.8$ & Initial heavy metal density (atoms/cc $10^{-24}$ ) \\
\hline 5.5 & Counter clock-wise rotation index $(0-5)$ & & \\
\hline 5.6 & Nuclide density sentinel $(0 / 1)$ & & \\
\hline 5.7 & Total number of fuel in rings in the assembly & & \\
\hline 5.8 & Fuel pin pitch $(\mathrm{cm})$ & & \\
\hline
\end{tabular}


Table 3.2. RCT Engineering Output Tables

\begin{tabular}{|c|l|}
\hline ID & \multicolumn{1}{c|}{ Table Header } \\
\hline 1 & Special Pin Material Densities \\
\hline 2 & Special Pin Location and Materials Assigned \\
\hline 3 & Heavy Metal Densities of Assemblies \\
\hline 4 & Mesh-cell Ordering and Region Assignment \\
\hline 5 & Region Average Number Density \\
\hline 6 & Neighboring Nodes and Surfaces \\
\hline 7 & Flux and Current of Assembly \\
\hline 8 & Coordinate of Fuel Pin Center \\
\hline 9 & Average Nuclide Densities, Burnup, and Fluence \\
\hline 10 & Flux at Center of Fuel Pin \\
\hline 11 & Power Density at Center of Fuel Pin \\
\hline 12 & Special Pin Densities, Burnup, and Fluence \\
\hline
\end{tabular}




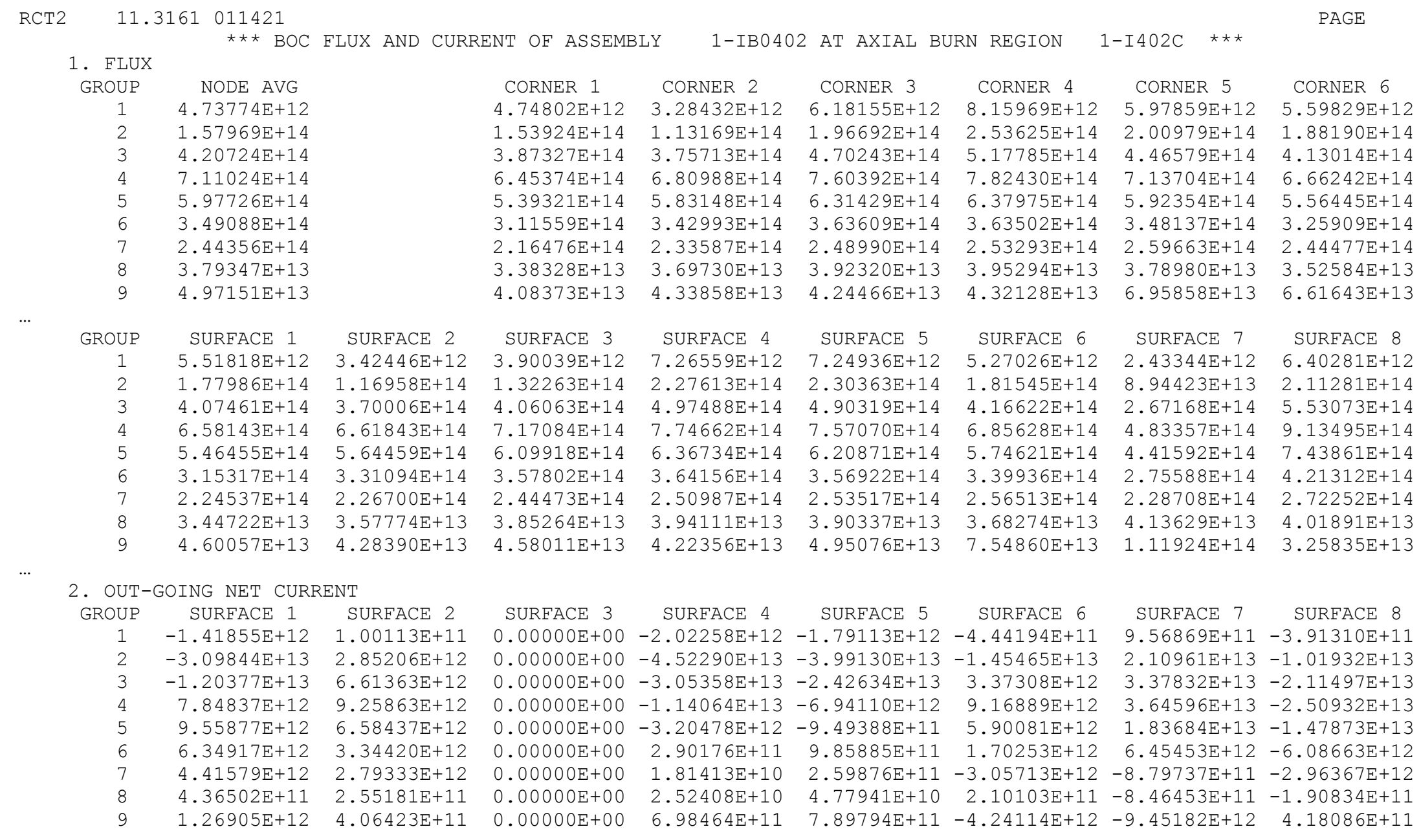

\section{Figure 3.4. An Example of the RCT Flux and Current Output Table}




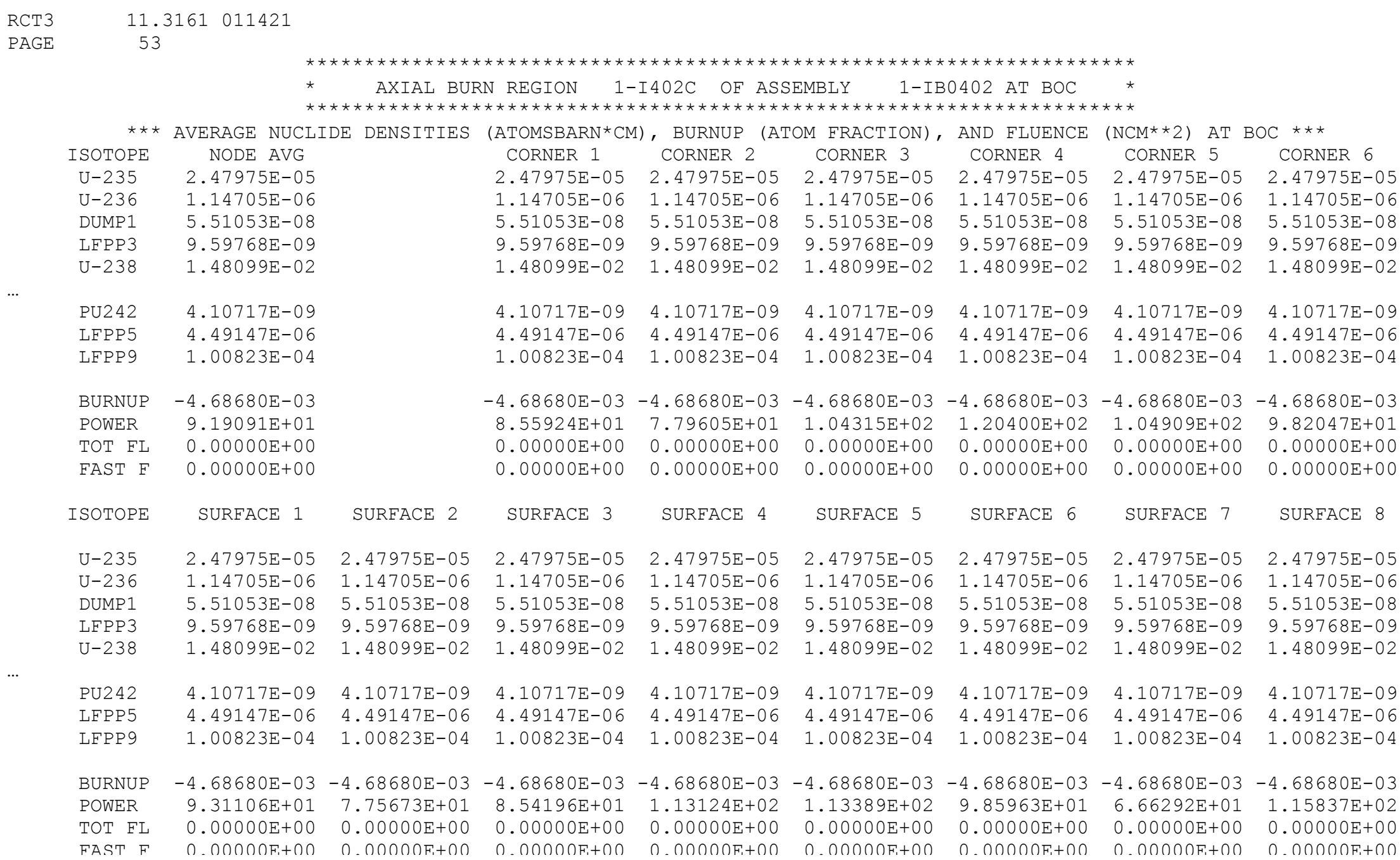

Figure 3.5. An Example of the Average Nuclide Densities, Burnup, and Fluence Table 
Table 3.3. RCT Identified Verification Tasks

\begin{tabular}{|c|c|c|}
\hline Category & \multicolumn{1}{|c|}{ Verification Tasks } \\
\hline \multirow{5}{*}{1} & Verify input details are accurate \\
a) All user input cards are obeyed correctly \\
b) The correct regions are identified for a given assembly \\
c) The correct compositions are selected for the identified regions \\
d) The axial plane details are consistent with the depleting materials \\
e) The mesh wise node averaged flux and partial currents are correct \\
f) The identified pin centers are correct
\end{tabular}


The goal of the verification work is to produce a set of verification problems that define the accuracy of RCT and document both the verification problem and result. In practice, a companion verification document will work through each verification test problem and selectively demonstrate how a subset of the tasks are satisfied by the verification problem and which output tables are being verified. Because the requirements document has not been approved, the RCT verification work cannot begin at this time. Moreover, based upon the experience with REBUS, additional tasks will likely end up being added to Table 3.3 as more details on how RCT is being used are exposed and thus both the requirements document and the verification report will have multiple revisions.

\subsection{Software Verification - SE2RCT}

As stated, SE2RCT is not included in Figure 2.2. SE2RCT is primarily the SUPERENERGY2 code [19] combined with the RCT interpolation scheme. SUPERENERGY-2 is focused on obtaining the steady state coolant temperature distribution in a ducted hexagonal rod bundle. In Figure 2.2, SUPERENERGY refers to SE2ANL which is SUPERENERGY-2 linked with a flux interpolation scheme based upon DIF3D-FD and other added features. In this manner, one can understand that the purpose of SE2RCT is similar to SE2ANL and thus there is some commonality in both the software and usage.

Overall, a lack of experience with SE2RCT makes it difficult to define the level of verification necessary for SE2RCT. SE2RCT is effectively a custom code derived from Superenergy-2 for the specific needs of the FIPD and any preceding programs that were similar. It has no functional purpose beyond that currently used in FIPD and there is no known manual that describes its usage. The lack of documentation and clarity on what parts of the SE2RCT output are being incorporated into FIPD makes it difficult to identify the engineering outputs of SE2RCT that are important. Three examples are given here to highlight the difficulties in creating a verification report.

The first example deals with the functional use of SUPERENERGY-2 code which was designed to solve the coolant temperature throughout the entire modeled reactor domain. In SE2RCT, a single assembly (typically an experimental assembly) from the original domain is targeted for analysis and only it and the six assemblies that surround it are solved. Adiabatic boundary conditions are applied on the outer edges of this seven assembly model. This aspect alone introduces accuracy concerns with regard to the consistency of the coolant temperature solution for a full core model compared with this seven assembly model that SE2RCT uses. Given the primary focus is only on the central assembly, it is likely not that important, but defining the impact on the accuracy of SE2RCT is part of the verification work.

In the second example, the fuel pin temperature calculation in SE2RCT makes several approximations and presents no documentation on how these approximations impact the accuracy. From the FIPD website, it appears as though SE2RCT output for the fuel pin temperature calculation is being provided to the user but it is not clear whether this is essential or ancillary information. As part of the work done this year, the impact of the approximations made in the fuel pin temperature calculation of SE2RCT was investigated and is discussed later in this report.

The final example is the flow rate input provided to SE2RCT. Measuring the individual assembly flow rate is not practical in a SFR and thus the EBRFLOW code was used to get the assembly flow rate for each depletion step given knowledge of the assembly orifice, the total measured pumping power, and the expected by-pass flow (flow between ducts that separate assemblies). The assembly flow rate input calculation can be considered part of PADB. For 
SE2RCT software verification, the flow rate information can be considered exact and thus be blindly trusted. The accuracy of the SE2RCT software would thus be bound by the accuracy of the input and the issue of inaccurate input is passed to the PADB software (or some intermediate scripting software) that prepares the input for SE2RCT. Given RCT is part of the verification and there is non-transparent hand off between RCT and SE2RCT, the concept that SE2RCT can be verified independent of the RCT usage in PADB and the manipulating software in between RCT and SE2RCT seems unwise. One could push further down this path on validation concerns. Given the way SE2RCT is being used on a single assembly, it is not clear how one would catch that a slightly incorrect flow rate or power level was being provided to SE2RCT for any of the seven assemblies and that slightly incorrect results would then be pulled into FIPD. In a full core SUPERENERGY-2 problem, one could, as an example, at least check that the total power being generated in the reactor is correct and that the power map matches that produced in ARC (a quick visual inspection). For SE2RCT, this requires knowledge of where all seven assemblies came from and then doing the cross check manually.

These examples tend to point out that the extraction of information from PADB to define the input of SE2RCT is a potential failure point for the SE2RCT software usage in FIPD. The same can be said for REBUS/RCT as the input there is also pulling from PADB. This would seem to indicate that the FIPD software QA envelope would need to include EBRFLOW and much of PADB as SE2RCT specific software verification will never account for the mistakes in the input preparation.

In the following sections, discussion of the work done with SE2RCT is provided and verification of some of the components is discussed. No requirements document was created in the current year as more experience with building a REBUS/RCT model from scratch are needed along with documentation of what output from SE2RCT is being used in FIPD.

\subsubsection{Identification and Correction of Coding Errors in SUPERENERGY-2}

SUPERENERGY-2 is not an Argonne software product and relative to DIF3D, it is one of the least tested codes in the ARC code system. This does not mean it is inaccurate, just that it is infrequently used and was not verified to the same level as the remaining ARC software. SE2ANL does not have a formal manual. SE2RCT also falls into this category as it is built upon SUPERENERGY-2 and there is little formal documentation of what it does. As part of other coordinated work, the SE2ANL code was being rebuilt as it was only connected to DIF3D-FD and some differences in the pin power distribution were observed when using the transport features of DIF3D-VARIANT compared with the diffusion limits of DIF3D-FD. There are other notable improvements being incorporated during that work to the methodology, but they are beyond the scope of this document. What is relevant is that the new implementation was compared directly to that of SE2ANL and two bugs were identified in the SUPERENERGY-2 code that were present in both SE2ANL and SE2RCT.

For the first bug, in the coolant temperature calculation part of the code, SE2ANL calculates conduction between adjacent edge-type subchannels and was found to use an incorrect value for the distance between those subchannels, despite pre-calculating the correct value prior to the problem sweep. Detailed investigation indicated that SUPERENERGY-2 was incorrectly comparing a global sub-channel index (over all assemblies) to a local sub-channel index (just the assembly being worked on). This meant that only the first assembly used the correct dimension while all of the other assemblies used the distance between the corner and edge subchannel for the 
distance between edge sub-channels. This occurred between lines 579 and 586 in calcxxx.f an excerpt of which is shown below.

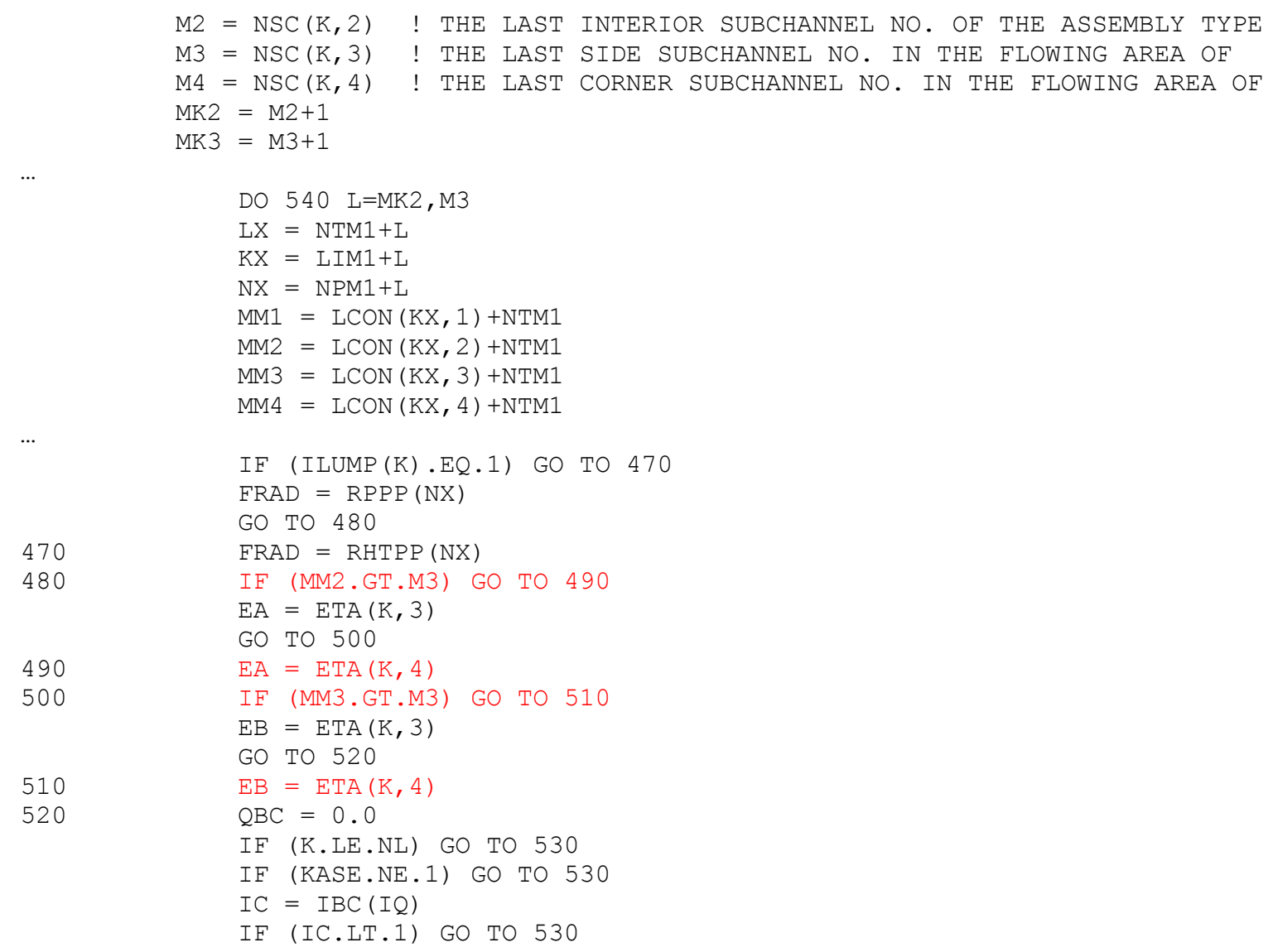

The above 540 loop only considers the edge sub-channels where MK2 is the first edge subchannel in the current assembly and M3 is the last edge sub-channel. The first interior sub-channel is simply 1 and there are always 6 corner sub-channels in the assembly.

The variable NTM1 is the offset to the current assembly sub-channels which are consecutively stored for all sub-channels of all assemblies. Thus MM1 to MM4 are the offsets to the connected sub-channels for the present sub-channel LX. If there are 10 assemblies each having 100 subchannels, then the NTM1 variable will have values of $0,99,199,299$, etc... for each assembly 1 , $2,3,4, \ldots$ In contrast, the variables MK2 and M3 will always be $<100$ as they are defined with respect to the assembly being worked on (i.e. not global quantities). The variable ETA(K,3) is for the edge to edge connection while $\operatorname{ETA}(\mathrm{K}, 4)$ is for the edge to corner connection.

Given the above discussion, it should be clear that the if statements at 480 and 500 will only be correct for the first assembly as the two if statements are comparing the global sub-channel position to the local sub-channel starting index. In all other cases, all edge-to-edge connections will be using $\operatorname{ETA}(\mathrm{K}, 4)$.

From the observed test case, the heat transfer between the edge and corners was increased because of this bug and thus the temperature distribution along the surface was flattened. Depending upon the pin diameter and pin pitch, one could just have easily made it the reverse such that heat transfer from the corner to the edge sub-channel was restricted and thus the opposite 
effect. The error occurred multiple times in the same subroutine and was easy to identify once when it became clear that global and local quantities should not be compared. For SE2RCT, the above error occurs in subroutine calc.f which is effectively identical to that shown in the excerpt above.

For the second bug, SE2ANL was observed to incorrectly calculate the Reynolds number in the Nusselt number calculation. The Nusselt number is written as

$$
\mathrm{Nu}=d_{1} \cdot \operatorname{Re}^{d_{2}} \operatorname{Pr}^{d_{3}}+d_{4}
$$

In the above equation, the four $\mathrm{d}$ values are provided by the user as input and are typically $0.025,0.08,0.08$, and 7.0 for most SFR applications. From inspection, it was determined that the $\mathrm{d} 4$ value dominates the magnitude of the coefficient because of the selection of $\mathrm{d} 1$. While there are several other correlations available for the Nu number, the preceding should make it clear that an incorrect $\mathrm{Re}$ number will cause an incorrect $\mathrm{Nu}$ and thus an incorrect heat transfer coefficient. The bug in this case was to artificially increase the magnitude of Re and thus the magnitude of the heat transfer coefficient. The bug was identified to occur around line 58 in findr.f an excerpt of which is shown below.

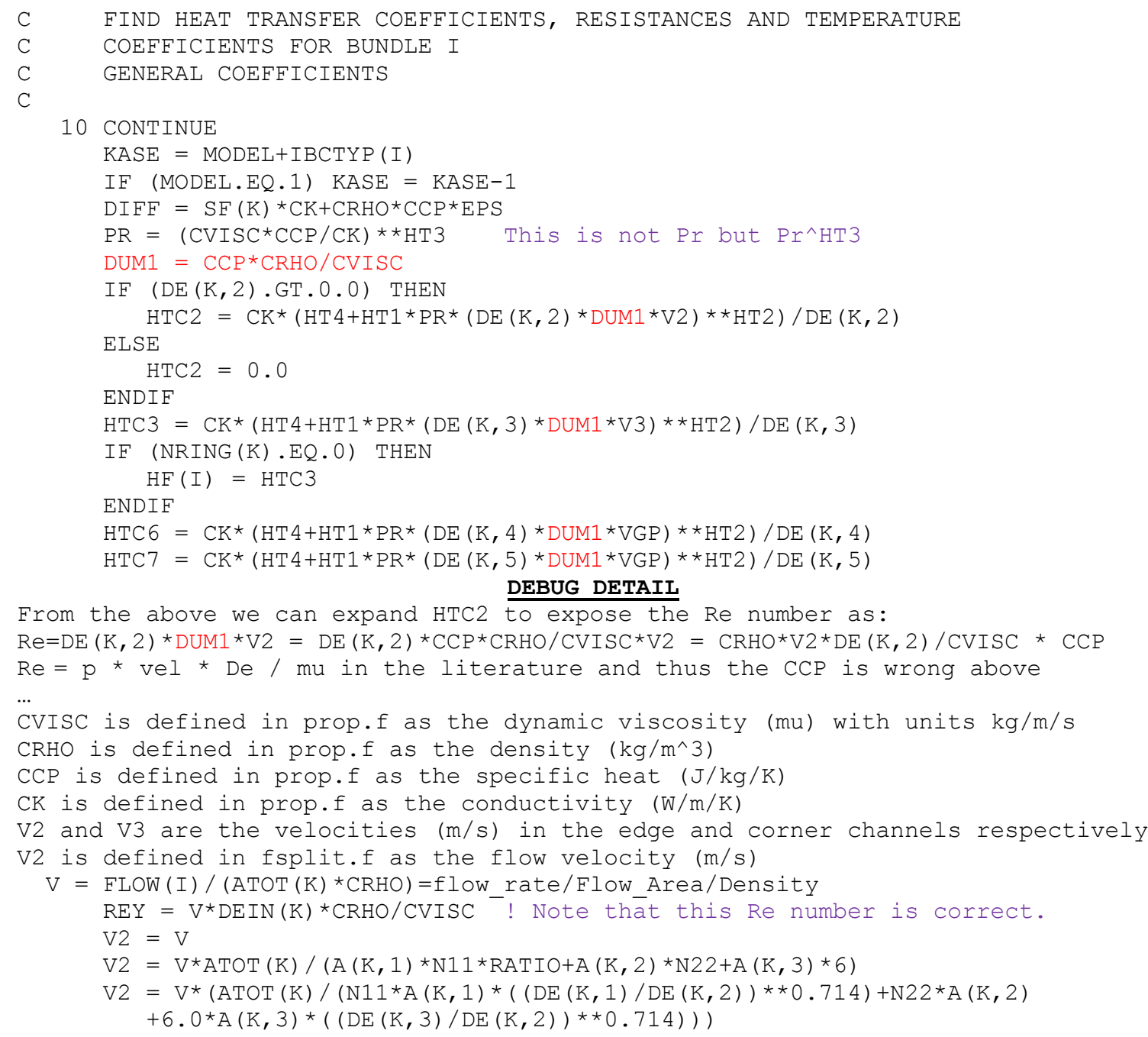


The excerpt shows a breakdown in the heat transfer coefficient and the debug detail provided at the bottom of the excerpt clearly indicates that Re number is wrong by a factor of $\mathrm{CCP}$ and thus the heat transfer coefficient HTC2 is wrong by a factor of CCPHT2 for typical input. Given CCP is on the order of 1000 and HT2 is typically user input (0.08), this means the heat transfer coefficient is approximately a factor of 2 higher than it should be. The same bug was present in the same subroutine of SE2RCT.

Two other bugs were found in the interface part of SE2ANL with SUPERENERGY-2 but since they are not part of SE2RCT, they are not included here. Both SE2ANL and SE2RCT were corrected to fix these bugs and the easiest way to study the impact is to look for differences in the coolant temperature maps. Figure 3.6 provides the SE2RCT temperature map for a single bundle before and after the bug is corrected. The upper left corner temperatures are highlighted to show the largest impact of the bug. It is important to note that a majority of the temperatures on the other surfaces are not impacted as much. This is an artifact of the type of calculation that SE2RCT is carrying out rather than the expected behavior. It is believed that the heat transfer coefficient bug is the dominant issue for this test case although separation of the two bugs was not investigated.

Because there are no real verification test cases for SUPERENERGY-2 and the SE2ANL cases are simply previous model Argonne staff have created, it is not clear whether these are the only bugs of note in SE2ANL or SE2ANL or whether others exist. Unlike DIF3D, REBUS, RCT or the other ARC software pieces, SUPERENERGY-2 presents a rather difficult software verification task.

\subsubsection{Verification Work - Pin calculation Routine}

One separable part of SE2ANL and SE2RCT is the fuel pin temperature calculation. Given the coolant temperature surrounding the pin, one should be able to calculate the fuel pin temperature details given the methodology in both codes is a simple 1D radial model. In the SE2ANL documentation, it is stated that a simplified fuel pin calculation will suffice for metal fuel because of the higher conductivity and thus lower temperature gradient over the fuel pin (all stated relative to oxide fuel). No supporting documentation was given to prove this aspect. Further, the metal fuel correlation was directly inserted into the analytic solution and a Newton-Raphson methodology was introduced to search for the temperature distribution.

This is all handled in the subroutine fpin.f of SE2ANL. This subroutine does not exist in SE2RCT as the fuel pin temperature calculation is handled with pint.f. It is noted that these two subroutines are quite different as the excerpt below shows. While the outer cladding temperature (TOD) is identical, the cladding midwall temperature (TMW) have rather different forms. Combined with the missing proof on the impact of the approximations and the recognition that this would be part of the verification work, time was spent to verify the fuel pin methodology where the results are compared against the SE2ANL result. 


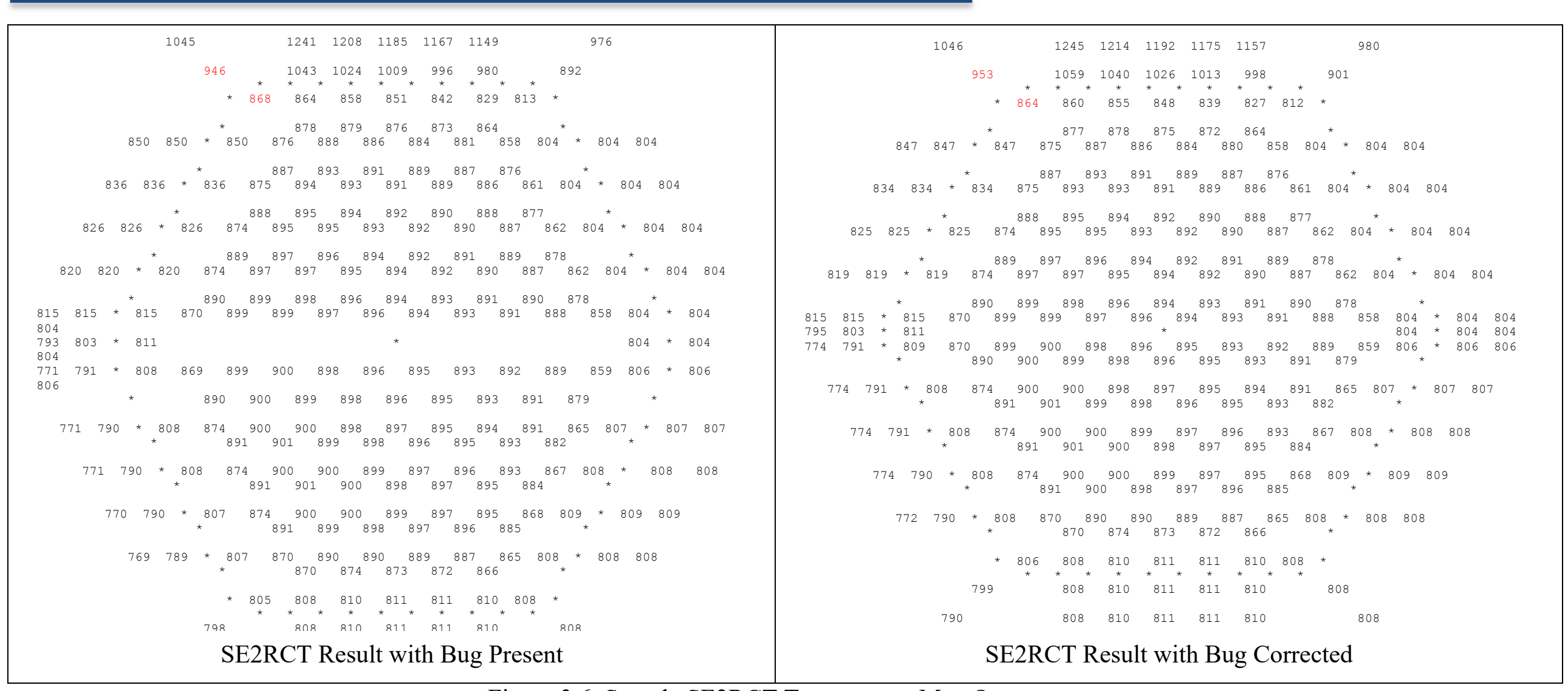

Figure 3.6. Sample SE2RCT Temperature Map Output 


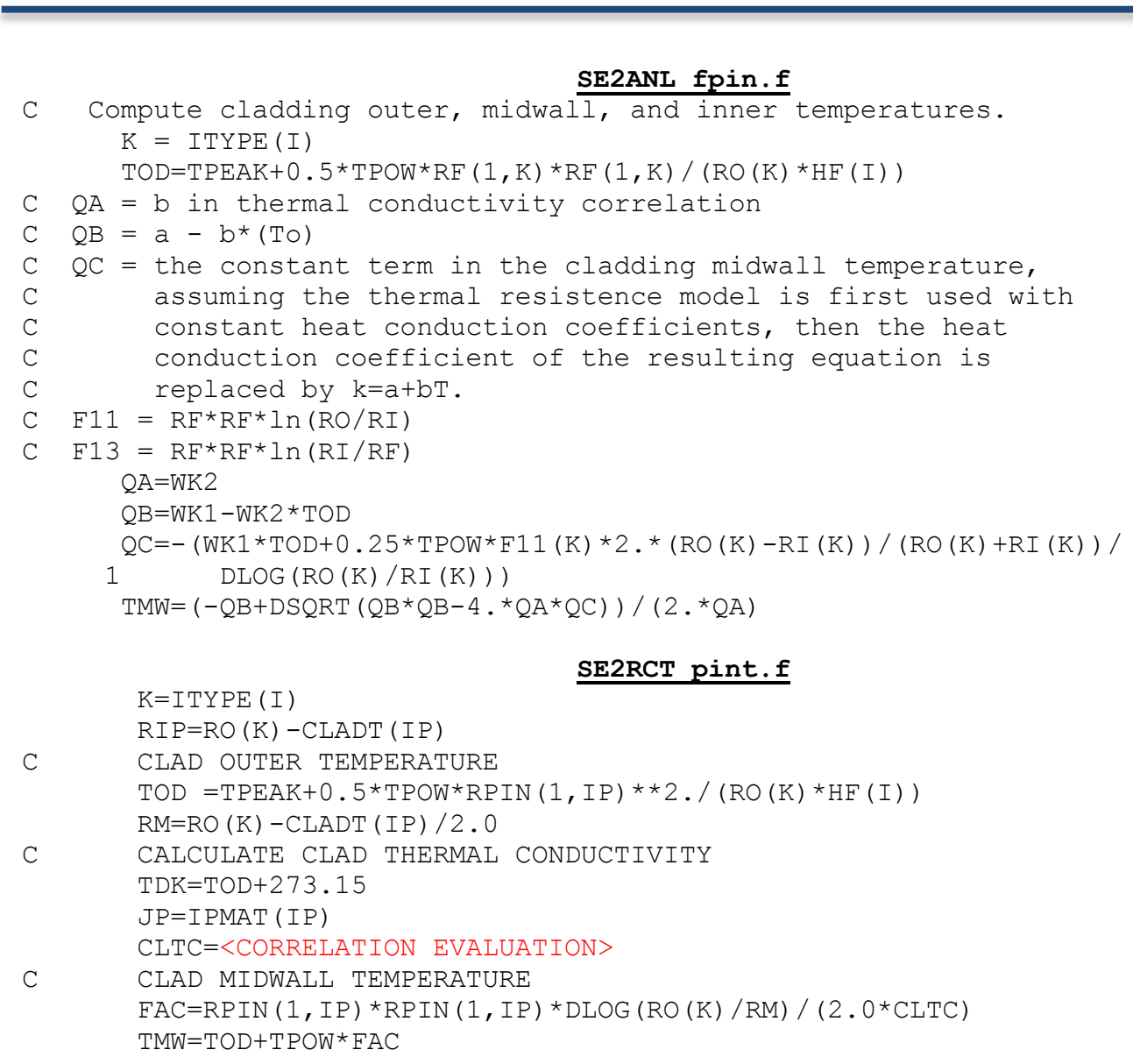

For the 1-D radial geometry, the differential equation has the form

$$
\frac{1}{r} \frac{d}{d r}\left(k \cdot r \cdot \frac{d T}{d r}\right)+q^{\prime \prime \prime}=0
$$

The analytic solution for a constant conductivity is

$$
T(r)=\frac{q^{\prime \prime \prime} \cdot r^{2}}{4 \cdot k}+\frac{C_{0} \cdot \ln (r)}{k}+C_{1} \text {. }
$$

Note that if the conductivity is not effectively constant, the above expression is not valid. Because the metal fuel thermal conductivity is a weak quadratic function with respect to temperature, the above holds in the limit of small mesh sizes and thus is valid for the present needs. For a series of $N$ meshes in the radial domain (fuel, gap, and cladding), the above equation holds for each mesh and thus the coefficients are unique to the total number of meshes and conductivity values. This produces $2 \cdot N$ unknowns to solve for in the stated problem. Two boundary conditions must be met at the interface between two adjacent meshes: continuity of the temperature and continuity of the temperature gradient. This produces $2 \cdot N-2$ boundary conditions. The central mesh must have $C_{0}=0$ and the outer most mesh can either be a temperature or a heat flux boundary condition to close the system. In both SE2ANL and SE2RCT, the temperature value of the outer surface of the cladding is used and thus it was used in this work.

This yields a simple matrix-vector system to be solved of the form $\overline{\bar{A}} \cdot \bar{C}=\bar{b}$. 
The simplifying algebra is not included for brevity and the matrix-vector system was programmed as a standalone code. To handle the temperature dependence of $\mathrm{k}$, the coefficient vector solve is wrapped in a search routine which updates the fuel and cladding conductivity with each progressive solution of the temperature field. Because the SE2ANL solution is a subset of this capability, the same routine was used to verify its consistency with the SE2ANL result and what is shown here is the externally calculated result (SE2ANL does not provide the radial detail).

Both the SE2ANL and SE2RCT methodology use a single mesh in the fuel region and a single mesh in the cladding. For comparison, results were generated using 2 and 4 meshes in the fuel and up to 2 meshes in the cladding. Figure 3.7 provides the results for the SE2ANL approach and using more fuel and cladding meshes.

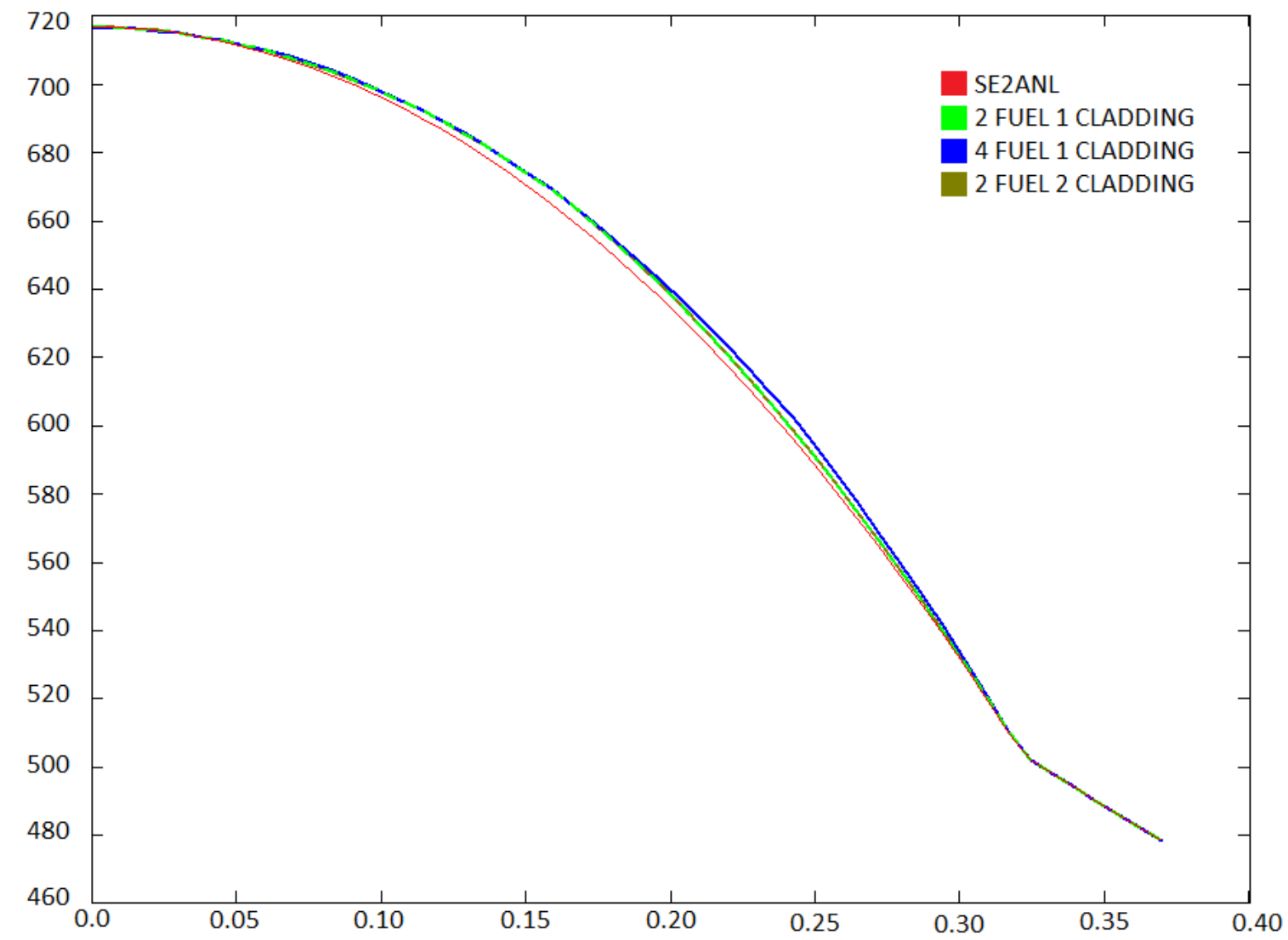

Figure 3.7. Results for the Fuel Pin Temperature Calculation.

As can be seen, as the number of fuel meshes is increased, the temperature solution tends to have a steeper gradient closer to the fuel/clad interface. The fuel centerline and clad midwall temperatures though are effectively the same which are tabulated in Table 3.4. The SE2ANL result produces the highest centerline temperature by a nearly trivial margin that is within the uncertainty on the fuel conductivity itself. Using more than 1 mesh in the cladding makes a negligible difference in the temperature solution while using 4 fuel meshes rather than 2 shows a progressive drop in the centerline temperature as the shape change indicates in Figure 3.7. 
This work verifies that the statement made in the SE2ANL documentation indicating a single fuel mesh would be sufficient is correct for the stated metal fuel. Further, it verifies that the Newton-Raphson methodology is working properly in SE2ANL and SE2RCT as a direct implementation of the SE2ANL approach with a much simpler search technique yielded the identical result to SE2ANL. Finally, the evaluation of the cladding conductivity at the outer surface temperature as done in SE2ANL and SE2RCT made a negligible difference in the temperature distribution.

Table 3.4. Fuel Centerline Results for the Fuel Pin Temperature Calculation

\begin{tabular}{|l|c|c|}
\hline & Centerline & Clad Midwall \\
\hline SE2ANL & 716.634 & 488.52 \\
\hline 2 FUEL 1 CLADDING & 716.548 & 488.52 \\
\hline 4 FUEL 1 CLADDING & 716.493 & 488.52 \\
\hline 2 FUEL 2 CLADDING & 716.551 & 488.51 \\
\hline
\end{tabular}

\subsection{Software Verification - Automated Code Verification and Documentation}

As highlighted in the preceding sections, a key element of software qualifications includes determination of software acceptance with respect to key critical characteristics relevant to the functional requirements of the software. To ensure software adequacy against these requirements, the software design process shall be documented, approved, and controlled. However, this process is time consuming and labor intensive.

NQA-1-2008/2009 requirements relevant to computer software include the following:

1. Software requirements shall be identified and documented and their selection reviewed and approved. Software requirements shall identify the operating system, function, interfaces, performance requirements, installation considerations, design inputs, and any design constraints.

2. Software Design: The software design shall be documented and shall define the design necessary to meet software requirements.

3. Implementation of the Software Design: The software design shall be implemented using documented programming standards and conventions.

4. Software Design Verification: Design verification shall be performed by a competent individual(s) other than those who developed and documented the design. Methods and results of design verification shall be documented. Acceptable verifications methods any one or combination of: design reviews, alternate calculations, and tests performed during development.

The software design verification steps are presented in more detail in Table 3.5. The steps identified in the table are often executed in a sequential manner and require manual preparation, review, and documentation from both the software designer and reviewer. The manual steps are presented in green in Figure 3.8.

A suitable model and its corresponding analytical solution are generated. Based on the analytical solution, an acceptance criterion is set. The computational model corresponding to the 
analytical set up is then prescribed and the solution compared against the analytical reference solution. This process can also be extended to validation (e.g. comparisons to experimental data). In some cases, where acceptance cannot be attained, a problem with the software may exist and would entail changes to the source code to fix bugs or errors. Performing acceptance testing is therefore critical to identify and correct software errors.

The process described can be extended to include software development activities where source code changes are implemented, and the software is retested.

Table 3.5: V\&V Test Suite Development Methodology

\begin{tabular}{c|l}
\hline Step & Activities \\
\hline Define test & $\begin{array}{l}\text { Develop test problem definition. The objective(s) of the test, key input } \\
\text { parameters, output metrics of interest, and acceptance criteria for the test } \\
\text { problem should be documented. Any initial conditions or clarifying } \\
\text { assumptions should also be stated. }\end{array}$ \\
$\begin{array}{c}\text { Develop } \\
\text { reference } \\
\text { solution }\end{array}$ & $\begin{array}{l}\text { Develop and document an analytical solution which may be based on a } \\
\text { system of first principles and implemented via a spreadsheet or hand } \\
\text { calculation. Simplified numerical solutions for more complex models are } \\
\text { also acceptable. The solution should meet the objectives of the test, utilize } \\
\text { the defined input parameters, and produce the relevant output metrics. } \\
\text { Any additional assumptions necessary to compute the solution should } \\
\text { also be clearly stated. At this stage, it may be necessary to choose } \\
\text { algorithms or solution schemes that match limitations of the software } \\
\text { (e.g. implementation of a linear approximation instead of exact solution } \\
\text { or use of particular meshing scheme). }\end{array}$ \\
\hline Create code & $\begin{array}{l}\text { Develop and document a model and corresponding code input and } \\
\text { generate a corresponding computational solution. The model should be } \\
\text { designed and built as per the problem definition in the first step. The } \\
\text { solution should meet the objectives of the test, utilize the defined input } \\
\text { parameters, and produce the relevant output metrics. Any additional } \\
\text { assumptions necessary to produce the solution should also be clearly } \\
\text { stated. It should be ensured that the assumptions and solution schemes } \\
\text { used in the computational and analytical solutions are consistent. }\end{array}$ \\
\hline $\begin{array}{c}\text { Compare } \\
\text { solutions }\end{array}$ & $\begin{array}{l}\text { Compare the analytical and computational solutions. Satisfaction of } \\
\text { acceptance criteria should be reviewed and documented. Deviations from } \\
\text { the acceptance criteria can be accepted if appropriate justifications are } \\
\text { developed. For cases that do not satisfy acceptance criteria, generate a } \\
\text { problem report. }\end{array}$ \\
\hline
\end{tabular}

Manual execution of this process requires the synchronization of the 3 steps: 1) generation of the reference solution, 2) software testing, and 3) documentation. The existence of the SQA program in place enforces that each component be reviewed, and its adequacy confirmed. This puts a significant burden on the developer and is not immune from human error. This process presented requires a significant effort from the code developers to maintain synchronization of the 
reference solution, the corresponding software and the documents capturing these verification and validation tests. Additionally, the process is repeated each time analytical inputs and/or the software inputs are updated. Each update requires a manual modification of the analytical input to update the analytical solution, the software input, the acceptance check and documentation of the software adequacy with the reference solution. This work can be generalized and automated such that any update in the test definition and acceptance criterion can be changed and be propagated automatically throughout the verification process for this test (i.e. generation of the reference solution, software results and documentation).

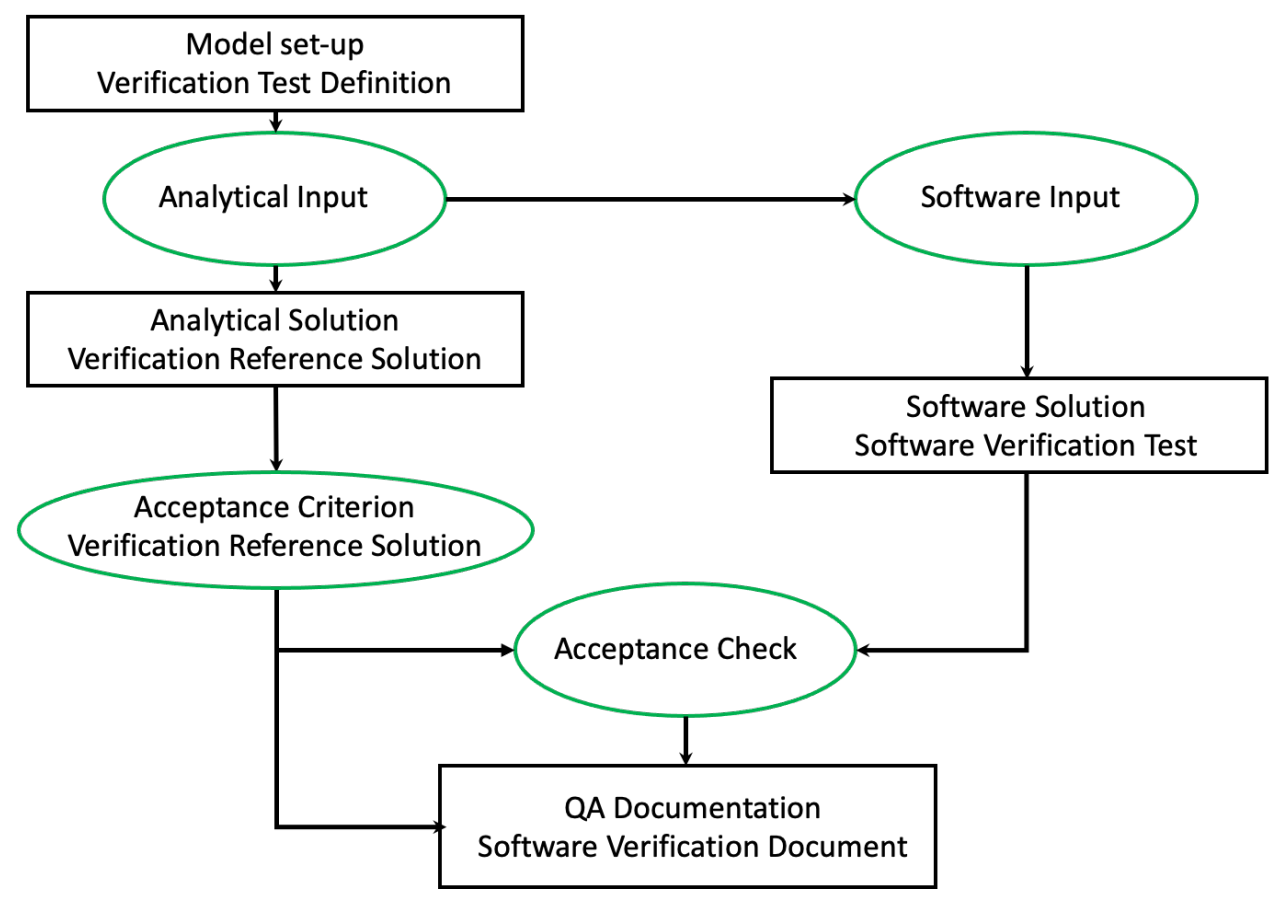

Figure 3.8. Current Manual Verification Process.

The proposed automated framework removes the need for individual input sets. A single verification input test is used by the reference solution generation, software solution, and QA documentation. The developer only needs to perform one manual check on the input (in addition to confirming the acceptance criterion). This is represented in the green boxes in Figure 3.9. From the centralized verification test input, the necessary information for each element of the verification test (reference solution, software solution, and Input/Output for the test documentation) is parsed automatically. Acceptance and output consistency checks are implemented during the automated "Acceptance Check" step, shown in purple in Figure 3.9. The number of manual checks is significantly reduced and burden on the developer falls in ensuring that only one set of inputs (instead of 3 ) contains the correct information. 


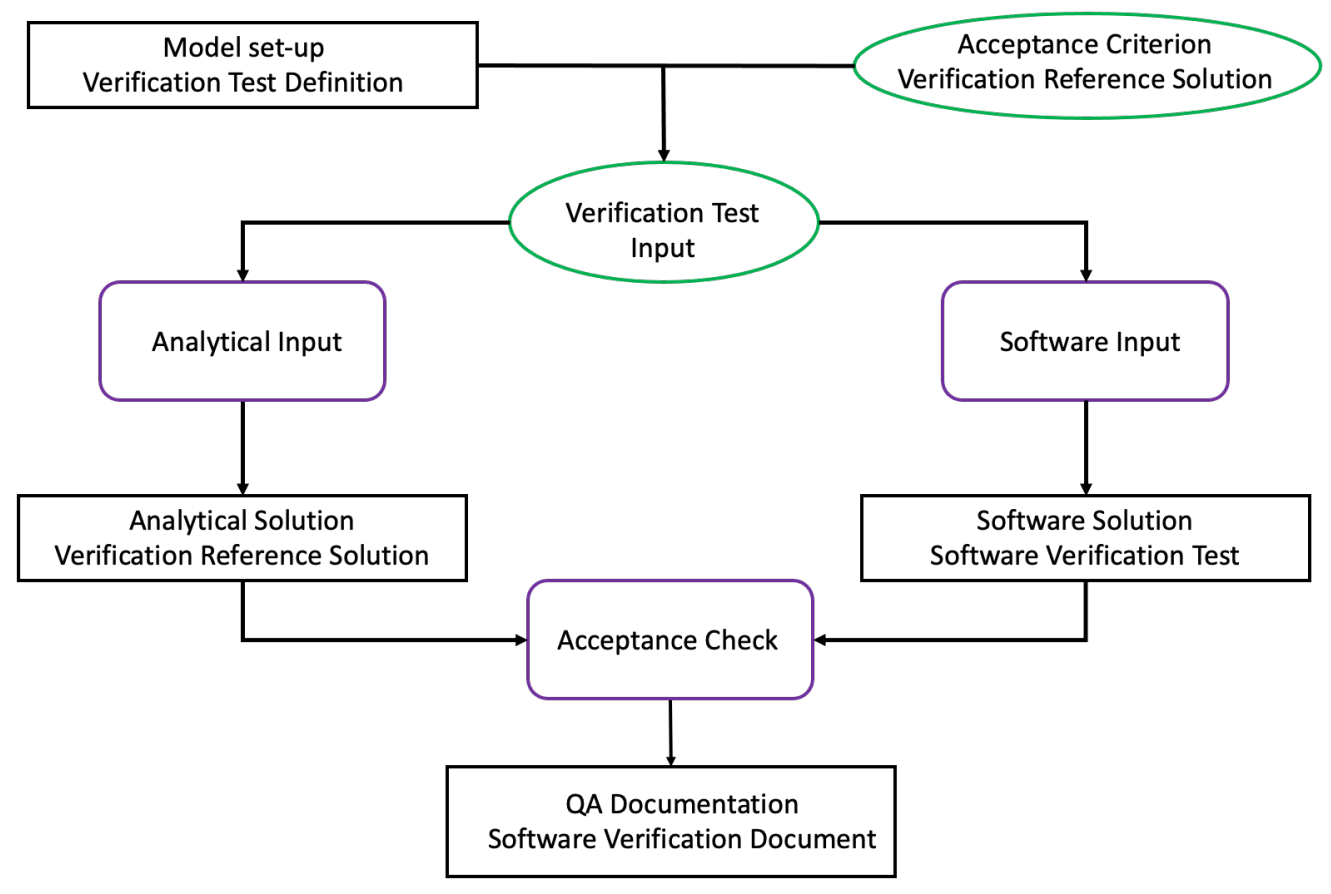

Figure 3.9. Proposed Verification Process

The development of this automated verification framework demonstrated the feasibility of a streamlined verification where reference solution, software results, comparison and documentation can be generated with minimum input from the software developer. Incorporating automation into the FIPD software QA process requires additional effort in the application of the code verification process. This represents however a promising process where FIPD data and software acceptance testing can be performed rapidly, and errors can be identified automatically. Future work for this task will expand the application of the automation of the verification and documentation process developed for as part of this effort to generalize its structure and expand the existing verification processes available for ARC.

\section{Summary}

The primary focus of this effort is to support the development and implementation of an SQA for the specific software used in FIPD, RCT and SE2RCT. In this report, a brief discussion on how this software is used in FIPD was provided along with uncertainties in the power normalization and number of depletion meshes. A "requirements" document was drafted which identifies the key features of RCT that are used by FIPD and thus which parts require software quality assurance. When approved, this document lays out the roadmap of verification work that needs to be completed and documented in a software verification report. All that work should be completed when the QA program is in place as it must be reviewed by an independent and knowledgeable person on RCT.

The creation of a SE2RCT requirements document was started, but a general lack of experience on SE2RCT and transparency on the information taken from SE2RCT and placed into FIPD prevented that document from being completed. As part of the rebuilding process of SUPERENERGY-2, two bugs were identified in SE2RCT this year which minorly impact the accuracy of past SE2RCT results. Additional work was also done to verify the fuel pin calculation 
methodology in SE2RCT as past documentation did not fully detail the impact of the approximations being made.

In addition to software quality assurance work for RCT and SE2RCT, an automated code verification framework is developed. The purpose of this framework is to streamline code verification and documentation while minimizing code developer input for repetitive tasks. The reduction of repeated input (between reference solution, software, and documentation input) throughout the SQA process reduces in turn potential for human error during the preparation of the supporting software quality records. Future work for this task will expand the application of the automation of the verification and documentation process developed for as part of this effort to generalize its structure and expand the existing verification processes available for ARC.

Future work will continue to focus on SE2RCT and the construction of verification problems that test out REBUS, RCT, and SE2RCT. This will be a crucial part of the verification work that ends up being required for SE2RCT and helps with gaining familiarity with the software usage in FIPD. 


\section{References}

1. A.M. Yacout, A. Oaks, W. Mohamed and K. Mo, "FIPD: EBR-II Fuels Irradiation \& Physics Database," ANL-ART-124. September (2017).

2. Walters, L.C., Seidel, B.R., Kittel, J.H., 1984. Performance of metallic fuels and blankets in liquid-metal fast breeder-reactors. Nucl. Technol. 65, 179-231.

3. Crawford, D., Porter, D., and Hayes, S., "Fuels for Sodium-cooled Fast Reactors: US Perspective," Journal of Nuclear Materials, vol. 371, pp. 202-231, 2007.

4. R. D. McKnight and B. J. Toppel, “The IFR Physics Analysis Database,” F6500-0019-AQ, Intra-Laboratory Memo, Argonne National Laboratory, September (1994).

5. R. D. McKnight, "Incorporation of the Measurement Data from Irradiated Fuel Samples into the MTG System at FCF", International Conference on the Physics of Nuclear Science and Technology, Islandia, Long Island, NY, October 5-8, (1998).

6. R. D. McKnight, "Validation of the REBUS-3/RCT Methodologies for EBR-II Core-Follow Analysis", ANL-IFR-153, September (1991).

7. R. W. Benedict and S. P. Henslee, 'The EBR-H Spent-Fuel Demonstration Project" Trans. Am Nucl. Soc., 77, p 75-76 (1997).

8. T. Benoit, "Quality Assurance Program Plan for SFR Metallic Fuel Data Qualification," ANL/NE-16/17 Rev. 2 (2019).

9. A. Yacout, K. Mo, A. Oaks, Y. Miao, T. Sofu, W. Mohamed, "FIPD: The SFR metallic fuels irradiation \& physics database," Nuclear Engineering and Design, Volume 380, (2021).

10. L. C. Just, H. Henryson II, A. S. Kennedy, S. D. Sparck, B. J. Toppel and P. M. Walker, "The System Aspects and Interface Data Sets of the Argonne Reactor Computation (ARC) System," ANL-7711, Argonne National Laboratory (1971).

11. C. H. Lee and W. S. Yang, "MC2-3: Multigroup Cross Section Generation Code for Fast Reactor Anaysis," ANL/NE-11-41, Revision 3, Argonne National Laboratory, August, 2018.

12. B. J. Toppel, “A User's Guide to the REBUS-3 Fuel Cycle Analysis Capability,” ANL-83-2, Argonne National Laboratory (1983).

13. W. S. Yang and M. A. Smith, "Theory Manual for the Fuel Cycle Analysis Code REBUS," ANL/NE-19/21 (2021).

14. K. L. Derstine, "DIF3D: A Code to Solve One-, Two-, and Three-Dimensional FiniteDifference Diffusion Theory Problems," ANL-82-64, Argonne National Laboratory (1984).

15. R. D. Lawrence, "The DIF3D Nodal Neutronics Option for Two- and Three-Dimensional Diffusion Theory Calculations in Hexagonal Geometry," ANL-83-1, Argonne National Laboratory, March 1983.

16. Palmiotti, G., Lewis, E. E., Carrico, C. B., "VARIANT: VARIational Anisotropic Nodal Transport for Multidimensional Cartesian and Hexagonal Geometry Calculation," Argonne National Laboratory ANL-95/40, 1995. 
17. M. A. Smith, E. E. Lewis, E. R. Shemon, "DIF3D-VARIANT 11.0, A Decade of Updates," ANL/NE-14/1 (2014).

18. M. A. Smith, C. H. Lee, and R. N. Hill, "GAMSOR: Gamma Source Preparation and DIF3D Flux Solution," ANL/NE-16/50 revision 1.0, June 28, 2017.

19. K. L. Basehore, N. E. Todreas, "SUPERENERGY-2: A Multi-Assembly Steady-State Computer Code for LMFBR Core Thermal-Hydraulic Analysis," PNL-3379 (1980).

20. M. A. Smith, "VARI3D \& PERSENT: Perturbation and Sensitivity Analysis," ANL/NE-13/8 Rev. 2 (2018).

21. W. S. Yang, "RCT - A Code to Reconstruct Pin Power and Burnup Characteristics from Nodal Calculations in Hexagonal Geometry," ANL-IFR-192, April 1993.

22. Won Sik Yang, "Sensitivity Theory for Reactor Burnup Analysis based on Depletion Perturbation Theory (DPT)," PhD Thesis Purdue University, May 1989.

23. J. Grudzinski, T. Moran, C. Grandy, "Supplement to the NUBOW-3D Manual," ANL/NE-15/9 (2015).

24. M. A. Smith and Aaron Oaks, Personal Communication, November 2020.

25. H.F. McFarlane, et al., "Benchmark Physics Tests in the Metallic-Fueled Assembly ZPPR15,” Nucl. Sci. Eng., 101, 137-152 (1989).

26. Argonne National Laboratory, "Reactors Design by Argonne National Laboratory," obtained from https://www.ne.anl.gov/About/reactors/frt.shtml in September (2019).

27. W. S. Yang and M. A. Smith, "RCT: REBUS Based Pin Power Reconstruction Using the DIF3D-Nodal and DIF3D-VARIANT Options," ANL/NE-14/15 (2014).

28. A. G. Nelson and M. A. Smith, "Verification of the DIF3D Software to Support Fast Reactor Analysis," ANL/NSE-20/3, Argonne National Laboratory (2020).

29. A. J. Brunett, L. Ibarra, T. H. Fanning, R. Hu "Improvements and Path Forward for Regulatory Acceptance of SAS4A/SASSYS-1," ANL/NSE-18/13, (2018).

30. N. L. C. Talbot, "User Manual for datatool bundle version 2.32," September (2019).

31. L. Lamport, "LATEX: a document preparation system.” Addison-Wesley Pub. Co. (1986). 


\section{Argonne}

\section{Nuclear Science and Engineering Division}

Argonne National Laboratory

9700 South Cass Avenue, Bldg. \#208

Argonne, IL 60439-4842

www.anl.gov

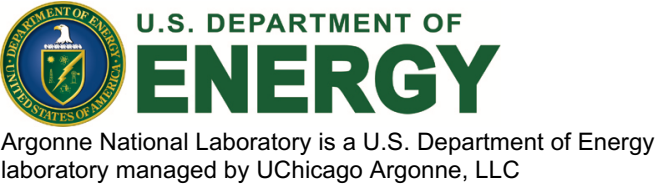

